\title{
Flutter Analysis of the Thermal Protection Layer on the NASA HIAD
}

\author{
Benjamin D. Goldman ${ }^{1}$ and Earl H. Dowell ${ }^{2}$ \\ Department of Mechanical Engineering and Materials Science, Duke University, Durham, NC, 27705 \\ Robert C. Scott ${ }^{3}$ \\ NASA Langley Research Center, Hampton, VA, 23681
}

\begin{abstract}
A combination of classical plate theory and a supersonic aerodynamic model is used to study the aeroelastic flutter behavior of a proposed thermal protection system (TPS) for the NASA HIAD. The analysis pertains to the rectangular configurations currently being tested in a NASA wind-tunnel facility, and may explain why oscillations of the articles could be observed. An analysis using a linear flat plate model indicated that flutter was possible well within the supersonic flow regime of the wind tunnel tests. A more complex nonlinear analysis of the TPS, taking into account any material curvature present due to the restraint system or substructure, indicated that significantly greater aerodynamic forcing is required for the onset of flutter. Chaotic and periodic limit cycle oscillations (LCOs) of the TPS are possible depending on how the curvature is imposed. When the pressure from the base substructure on the bottom of the TPS is used as the source of curvature, the flutter boundary increases rapidly and chaotic behavior is eliminated.
\end{abstract}

\section{Nomenclature}

$\mathrm{a}=$ plate length

$a_{n} \quad=$ modal coordinate of Kevlar/general plate

$a_{\infty} \quad=$ speed of sound

$\mathrm{b}=$ plate width

$b_{r} \quad=$ modal coordinate of Nextel

$c_{n m}=$ Fourier coefficient for the imposed-pressure geometry

$\mathrm{C}=$ damping matrix

$\mathrm{D} \quad=$ plate bending rigidity

$\mathrm{E} \quad=$ Young's modulus

$\mathrm{F} \quad=$ Airy stress function

$f_{j} \quad=$ modal coordinate of the Airy function expansion

$\mathrm{H} \quad=$ rise height of a curved plate

$\mathrm{h} \quad=$ plate thickness

$k \quad=$ spring constant

$\mathrm{K}=$ stiffness matrix

$\mathrm{M} \quad=$ mass matrix

$\mathrm{m} \quad=$ mass/area of plate

\footnotetext{
${ }^{1}$ Graduate Student, Dept. of Mechanical Engineering and Materials Science, Box 90300 Hudson Hall, Durham, NC 27708, Student Member AIAA

${ }^{2}$ William Holland Hall Professor, Dept. of Mechanical Engineering and Materials Science, Box 90300 Hudson Hall, Durham, NC 27708, Honorary Fellow AIAA

${ }^{3}$ Aerospace Engineer, Aeroelasticity Branch, NASA Langley Research Center, Member AIAA
} 


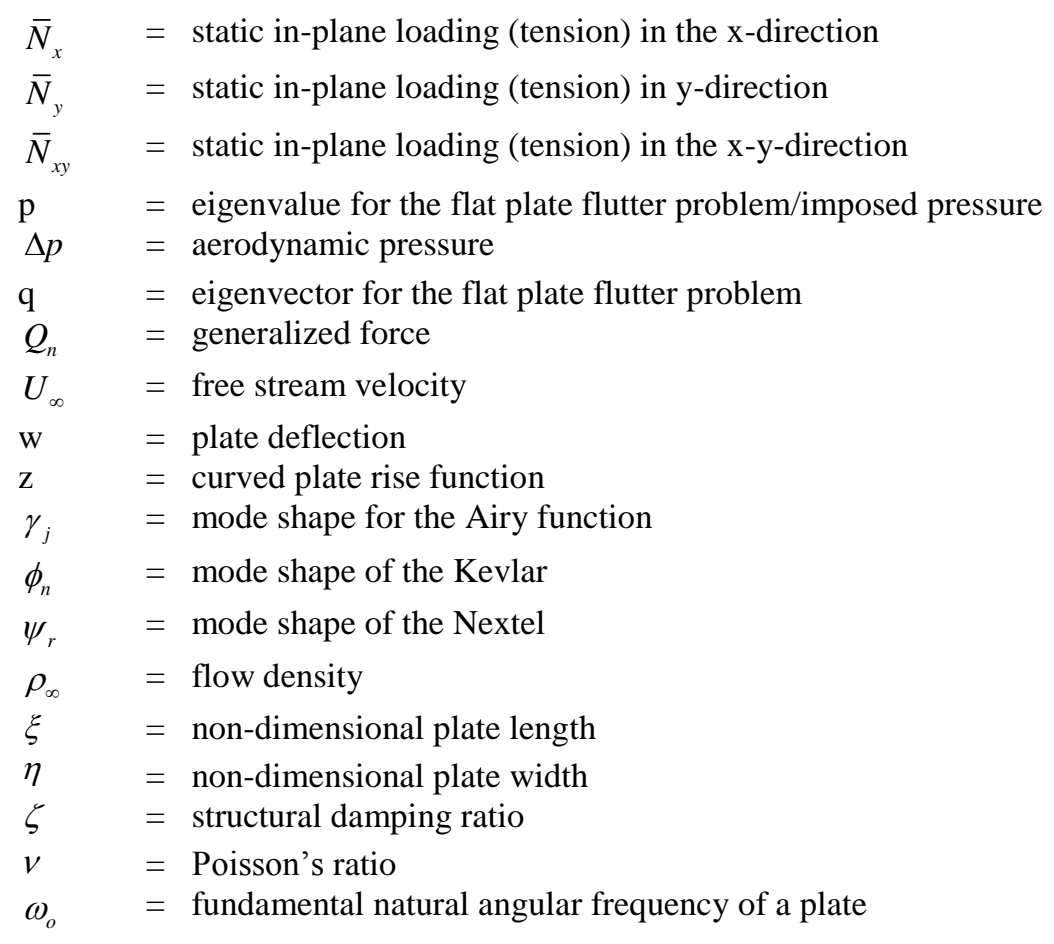

\section{Introduction}

$\mathrm{T}$ he purpose of the following analysis is to characterize the flutter behavior of the proposed thermal protection layer (TPS) on the NASA Hypersonic Inflatable Aerodynamic Decelerator (HIAD). The TPS is composed of several layers of different materials, each with unique physical properties. At certain flight conditions, characterized by flow velocity and density, interactions between aerodynamic forces and the structure may cause a dynamic instability known as aeroelastic flutter. Since this instability could damage the TPS and possibly expose the HIAD to the high temperature flow of re-entry, it is important to determine the conditions in which flutter may occur.

While the aeroelastic behavior of the HIAD of great interest, its complex geometry makes it difficult to analyze, especially with respect to flutter. Thus, as a first step, the present analysis is based on the current NASA wind-tunnel tests of rectangular TPS articles ${ }^{1}$, which are mounted on metallic tiles and exposed to hypersonic flow. A flutter analysis of this geometric shape is less complex, and it may help explain why oscillations could be observed during testing. While flutter is a possibility, excitation of a base structural component or turbulent boundary layer fluctuations cannot yet be ruled out as sources of oscillation. In any case, this investigation is an important foundation for the eventual consideration of the aeroelastic behavior of the TPS in its full flight configuration.

\section{Theoretical Models}

Several structural models have been developed for the rectangular test article, including both flat and curved plate models. The test article is a multi-layer structure consisting of Nextel 400-BF20, Pyrogel 6650, and Aluminized Kapton Kevlar Laminate. Curvature was estimated based on photographs of the test samples in an earlier AIAA report by Hughes, et $\mathrm{al}^{2}$. In all cases, piston theory is used for the aerodynamic pressure. This linear unsteady theory assumes the induced aerodynamic pressure is a result of the instantaneous plate deflection only, and thus memory effects are ignored.

\section{A. Linear Flat Plate Model}

The first proposed structural model is a two-dimensional linear system consisting of two flat plates separated by a spring-damper, shown in Figure 1. The Nextel and Kevlar are the flexible plate members while the inner Pyrogel layer is a spring-damper. Since the Pyrogel is relatively thick and sponge-like, it can be compressed as a result of deflection of both the Nextel and Kevlar. The model assumes that the Nextel and Kevlar are connected to the Pyrogel at every point along the length of the sample, which is an approximation whose validity depends on how the layers are stitched together. 
In-plane loading (tension) will also be considered, since some tension may be applied in the wind-tunnel test. A uniform uni-directional supersonic flow along the plate length with velocity $U$ is also present. Simply supported boundaries are shown here; however, other boundary conditions such as clamped and free can be imposed and will be discussed later. Note that by setting the spring stiffness and damping ratio of the Pyrogel to zero, the system decouples and reduces to a single Nextel (or Kevlar) plate which may also be of interest. By increasing the bending stiffness of the Kevlar to a large value, the system becomes a single Nextel plate on a Pyrogel foundation. Flutter points and natural frequencies were calculated for all of these configurations and are discussed in the results section.

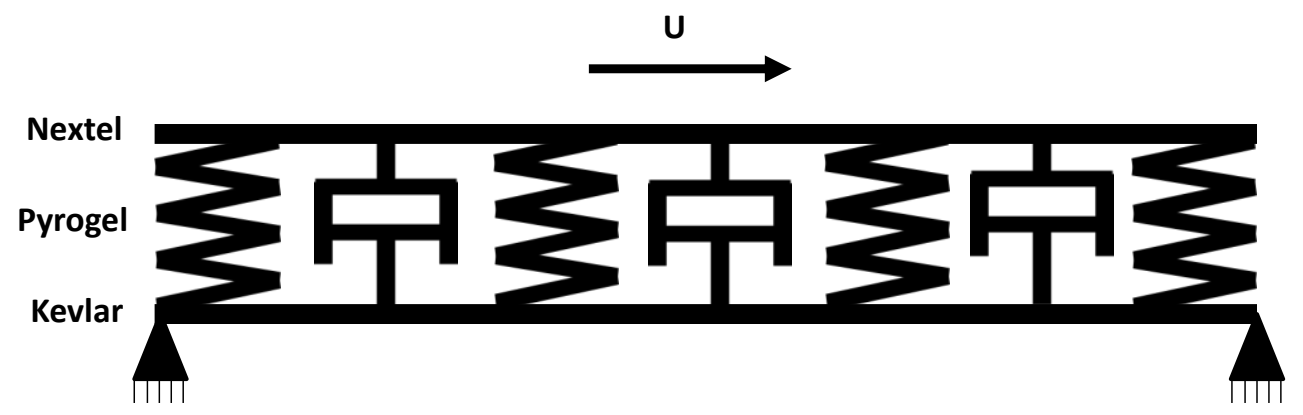

Figure 1. Flat plate model of the TPS system with simply supported boundary conditions and flow velocity $U$.

\section{Governing Equations}

A classical Lagrangian energy approach was used to derive the equations of motion for this system. The general formulation is provided below, and the detailed expressions for the modal equations can be found in the appendix. The total potential energy of the system is:

$$
U_{T O T}=U_{B}^{K E V}+U_{B}^{N E X}+U^{P Y R}
$$

With

$$
\begin{aligned}
U_{B}^{K E V}= & \frac{D^{K E V}}{2} \int_{0}^{b} \int_{0}^{a}\left[\left(\frac{\partial^{2} w^{K E V}}{\partial x^{2}}\right)^{2}+\left(\frac{\partial^{2} w^{K E V}}{\partial y^{2}}\right)^{2}+2 v^{K E V}\left(\frac{\partial^{2} w^{K E V}}{\partial x^{2}}\right)\left(\frac{\partial^{2} w^{K E V}}{\partial y^{2}}\right)+2\left(1-v^{K E V}\right)\left(\frac{\partial^{2} w^{K E V}}{\partial x \partial y}\right)^{2}\right] d x d y \\
& +\frac{\bar{N}_{x}^{K E V}}{2} \int_{0}^{b} \int_{0}^{a}\left(\frac{\partial w^{K E V}}{\partial x}\right)^{2} d x d y+\frac{\bar{N}_{y}^{K E V}}{2} \int_{0}^{b} \int_{0}^{a}\left(\frac{\partial w^{K E V}}{\partial y}\right)^{2} d x d y \\
U_{B}^{N E X}= & \frac{D^{N E X}}{2} \int_{0}^{b} \int_{0}^{a}\left[\left(\frac{\partial^{2} w^{N E X}}{\partial x^{2}}\right)^{2}+\left(\frac{\partial^{2} w^{N E X}}{\partial y^{2}}\right)^{2}+2 v^{N E X}\left(\frac{\partial^{2} w^{N E X}}{\partial x^{2}}\right)\left(\frac{\partial^{2} w^{N E X}}{\partial y^{2}}\right)+2\left(1-v^{N E X}\right)\left(\frac{\partial^{2} w^{N E X}}{\partial x \partial y}\right)^{2}\right] d x d y \\
& +\frac{\bar{N}_{x}^{N E X}}{2} \int_{0}^{b} \int_{0}^{a}\left(\frac{\partial w^{N E X}}{\partial x}\right)^{2} d x d y+\frac{\bar{N}_{y}^{N E X}}{2} \int_{0}^{b} \int_{0}^{a}\left(\frac{\partial w^{N E X}}{\partial y}\right)^{2} d x d y \\
U^{P Y R}= & \frac{k^{P Y R}}{2} \int_{0}^{b} \int_{0}^{a}\left(w^{N E X}-w^{K E V}\right)^{2} d x d y
\end{aligned}
$$

Note that only bending potential energy is used here. An assumption has been made that the plate layers do not sufficiently stretch during deflection, which is the case for flat plates with oscillations substantially less than the thickness ${ }^{3}$. For larger deflections, in the case of initial/imposed curvature or post-flutter limit cycle oscillations, the 
plate material may stretch, which would require the addition of a stretching potential energy. This addition will be important for the curved plate models in the next sections. The total kinetic energy for this system is:

$$
T_{T O T}=T^{K E V}+T^{N E X}+T^{P Y R}
$$

With

$$
\begin{gathered}
T^{K E V}=\frac{m^{K E V}}{2} \int_{0}^{b} \int_{0}^{a}\left(\frac{\partial w^{K E V}}{\partial t}\right)^{2} d x d y \\
T^{N E X}=\frac{m^{N E X}}{2} \int_{0}^{b} \int_{0}^{a}\left(\frac{\partial w^{N E X}}{\partial t}\right)^{2} d x d y \\
T^{P Y R}=\frac{m^{P Y R}}{2} \int_{0}^{b} \int_{0}^{a}\left(\frac{\partial w^{N E X}}{\partial t}+\frac{\partial w^{K E V}}{\partial t}\right)^{2} d x d y
\end{gathered}
$$

The Lagrangian is:

$$
L=T_{T O T}-U_{T O T}
$$

The deflection of both the Kevlar and Nextel can be expanded using the following modal series:

$$
\begin{aligned}
& w^{K E V}(x, y, t)=\sum_{n} a_{n}(t) \varphi_{n}(x, y) \\
& w^{N E X}(x, y, t)=\sum_{r} b_{r}(t) \psi_{r}(x, y)
\end{aligned}
$$

The modal functions $\varphi_{n}$ and $\psi_{r}$ are the products of beam modes that depend on the boundary conditions of the problem. Only one mode is retained in the spanwise direction, which is a valid assumption since the plate is flat and the flow is only along the plate length. Substituting Eq. (1.6) into Eq. (1.5) results in a Lagrangian in terms of the two modal coordinates $a_{n}$ and $b_{r}$. Lagrange's equations for the problem are:

$$
\begin{aligned}
& \frac{d}{d t}\left(\frac{\partial L}{\partial \dot{a}_{n}}\right)-\frac{\partial L}{\partial a_{n}}+Q_{n}=0 \\
& \frac{d}{d t}\left(\frac{\partial L}{\partial \dot{b}_{r}}\right)-\frac{\partial L}{\partial b_{r}}+Q_{r}=0
\end{aligned}
$$

The generalized force terms $Q_{n}$ and $Q_{r}$ incorporate the aerodynamic pressure and any structural damping that may be present. In this analysis, the aerodynamic pressure is given by the piston theory approximation for supersonic/hypersonic flow:

$$
\Delta p=\rho_{\infty} a_{\infty}\left[U_{\infty} \frac{\partial w}{\partial x}+\frac{\partial w}{\partial t}\right]
$$

The aerodynamic pressure is only applied directly to the outer Nextel, so the Kevlar is not exposed to the flow. The stability of the system can be studied by further expanding the time dependent modal coordinates as follows: 


$$
\begin{aligned}
& a_{n}=\hat{a}_{n} e^{p t} \\
& b_{r}=\hat{b}_{r} e^{p t}
\end{aligned}
$$

The variable $p$ is a complex number which characterizes both system damping and frequency. Substituting Eq. (1.9) into the equations of motion derived from Eq. (1.7) produces the following eigenvalue problem for stability of the system:

$$
\left(p^{2} M+p C+K\right)\{q\}=\{0\}
$$

The quantities $\mathrm{M}, \mathrm{C}$, and $\mathrm{K}$ are the mass, damping, and stiffness matrices, respectively. This eigenvalue problem is solved using the P-K method, whereby the eigenvalues are calculated for a given frequency until convergence is attained, and then stability is determined by observing any sign changes that occur in the damping, all the while increasing a flow parameter in small increments.

\section{B. Parabolic Curvature Model}

This three-dimensional nonlinear structural model accounts for any small amount of curvature that the TPS may exhibit when tensioned across the rigid tile. The test configuration from which this model is based is shown in Figure 2, and the model representation is given in Figure 3. Visual inspection of the test article indicates some (approximately) parabolic curvature due to the edge restraint bracket. The model assumes there is a space between the center of the bottom Kevlar layer and the center of the tile which is equal to the curvature rise height $(\mathrm{H})$. The curvature is assumed to be a parabolic function in both the streamwise and spanwise directions, although results will also be computed for the case with streamwise curvature only. The maximum rise height of the tensioned TPS will be estimated as on the order of five times the thickness of the Nextel, which is approximately $0.5 \mathrm{~cm}$. Calculations will also be performed for a range of smaller rise heights, since interesting flutter behavior may arise in this regime. Note that the flutter amplitudes of curved plates are on the order of the rise height, rather than the plate thickness ${ }^{4}$, as is the case for flat plates. This is why nonlinear large-deflection theory is required.

Additionally, the piston theory aerodynamic pressure in Eq. (1.8) must be modified to account for the presence of curvature, resulting in an additional static loading term proportional to the initial plate slope. This static load is critical in determining the flutter boundary. When the curvature is set to zero, this model reduces down to a threedimensional nonlinear version of the previous two-dimensional linear flat plate model with standard piston theory aerodynamics.

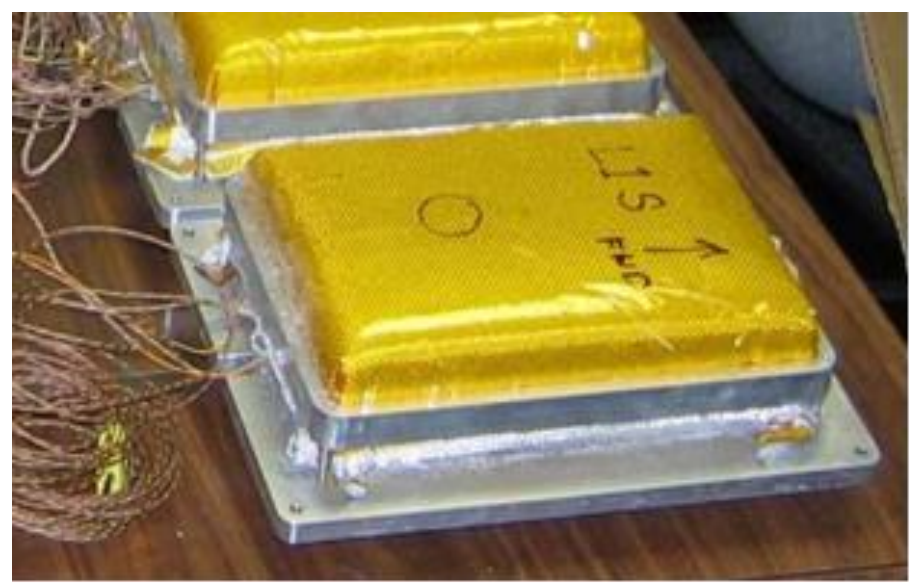

Figure 2. TPS test article tensioned across rigid metallic tile, exhibiting streamwise and spanwise curvature ${ }^{2}$. 


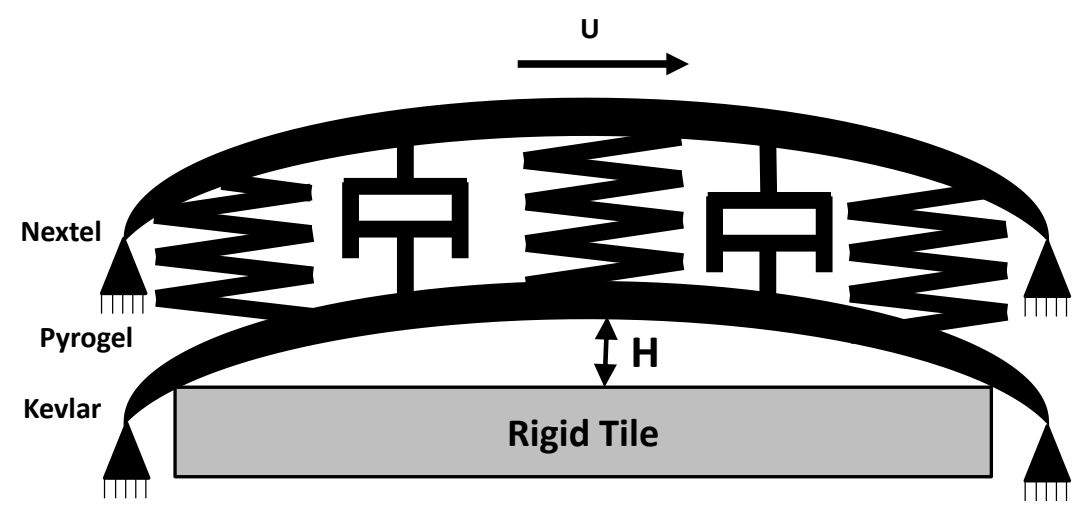

Figure 3. Theoretical structural model of the TPS article with initial parabolic curvature.

\section{Governing Equations}

The equations of motion for this system were derived using the Airy stress function with stress boundary conditions for the in-plane deformation. The bracket shown in Figure 2 may apply stress at the boundaries, but it is likely smaller than the stress due large amplitude out-of-plane bending. The bracket also cannot enforce a condition of vanishing slope at the boundaries, since a small space exists between the material and the bracket. These two observations imply stress-free boundary conditions, although the effect of applied stresses will be considered as well. The out of plane boundary conditions will be approximated as simply-supported, since some degree of rotation is possible within the space between the bracket and TPS. This configuration will be a lower-bound for flutter, since simply supported edges give rise to a lower flutter boundary than clamped edges (see Table 7).

The governing equations for the TPS with parabolic curvature were derived using the same Lagrangian approach for the flat plate analysis, with some modifications to each individual plate layer. The total potential energy for a single plate is now:

$$
U_{1-\text { plate }}=U_{B}+U_{S}
$$

The bending potential energy is the same as Eq. (1.2), and the stretching potential energy is:

$$
U_{s}=\frac{1}{2 E h} \int_{0}^{b} \int_{0}^{a}\left[\left(\frac{\partial^{2} F}{\partial x^{2}}\right)^{2}+\left(\frac{\partial^{2} F}{\partial y^{2}}\right)^{2}-2 v\left(\frac{\partial^{2} F}{\partial x^{2}}\right)\left(\frac{\partial^{2} F}{\partial y^{2}}\right)+2(1+v)\left(\frac{\partial^{2} F}{\partial x \partial y}\right)^{2}\right] d x d y
$$

Where $\mathrm{F}$ is the Airy stress functions defined by:

$$
\frac{\partial^{2} F}{\partial x^{2}}=N_{y} \quad \frac{\partial^{2} F}{\partial y^{2}}=N_{x} \quad-\frac{\partial^{2} F}{\partial x \partial y}=N_{x y}
$$

The quantities $N_{x}, N_{y}$, and $N_{x y}$ are the in-plane stress resultants. The Airy function also satisfies the following equation of strain compatibility, which is modified for the case of initial curvature:

$$
\frac{\nabla^{4} F}{E h}=\frac{\partial^{2}}{\partial x \partial y}(w+z) \frac{\partial^{2}}{\partial x \partial y}(w+z)-\left(\frac{\partial^{2} z}{\partial x \partial y}\right)^{2}-\frac{\partial^{2}}{\partial x^{2}}(w+z) \frac{\partial^{2}}{\partial y^{2}}(w+z)+\frac{\partial^{2} z}{\partial x^{2}} \frac{\partial^{2} z}{\partial y^{2}}
$$


The function $\mathrm{z}$ is the parabolic plate rise function defined by:

$$
\mathrm{z}=16 \mathrm{H} \frac{x}{a}\left(1-\frac{x}{a}\right) \frac{y}{b}\left(1-\frac{y}{b}\right)
$$

The modification to the piston theory aerodynamic model, to account for plate rise, is given by:

$$
\Delta p=\rho_{\infty} a_{\infty}\left[U_{\infty} \frac{\partial w}{\partial x}+\frac{\partial w}{\partial t}+U_{\infty} \frac{\partial z}{\partial x}\right]
$$

Eq. (1.14) along with Eq. (1.12) and Eq. (1.11) comprise the modified energy formulation of Von Karman's large deflection theory. The solution procedure begins by again expanding all time-dependent functions into modal series:

$$
\begin{aligned}
& w(x, y, t)=\sum_{n} a_{n}(t) \phi_{n}(x, y) \\
& F(x, y, t)=F_{p}+\sum_{j} f_{j}(t) \gamma_{j}(x, y)
\end{aligned}
$$

Again, both modal functions must satisfy the boundary conditions. The particular solution $F_{p}$ is the solution to the homogenized version of Eq. (1.14), and is associated with the static loading of the plate ${ }^{4}$ :

$$
F_{p}=\frac{\bar{N}_{y} x^{2}}{2}+\frac{\bar{N}_{x} y^{2}}{2}-\bar{N}_{x y} x y
$$

The quantities $\bar{N}_{x}, \bar{N}_{y}$, and $\bar{N}_{x y}$ also satisfy stress boundary conditions. For stress free boundaries, these quantities are zero. When stress is imposed at the boundaries, it is assumed that no shear stress is applied. A solution to the full strain compatibility condition is obtained via Galerkin integration, which allows the stress coordinate $f_{j}$ to be written in terms of the deflection coordinate $a_{n}$. The detailed equations of motion for a single plate are given in the appendix.

\section{Imposed Pressure Model}

In this analysis, the shape of the TPS is assumed to be the same as the metallic tile substructure. Unlike the previous model, this configuration has a flat middle surface with parabolic curvature only at the edges. The experimental configuration from which the model is based is shown in Figure 4, and the theoretical model is shown in Figure 5. This model also assumes that when the TPS is secured across the metallic substructure, a static pressure is imposed on the Kevlar. This is the pressure required to generate the initial geometry of the TPS, which can be solved via a static inverse problem. This model can also be interpreted as a three-dimensional, nonlinear version of the flat plate model with an additional generalized force due to the imposed pressure. Note that the infinite rigidity of the tile substructure will not be considered. That would require enforcing the condition that there be no deflection at every point where the TPS contacts the tile. 


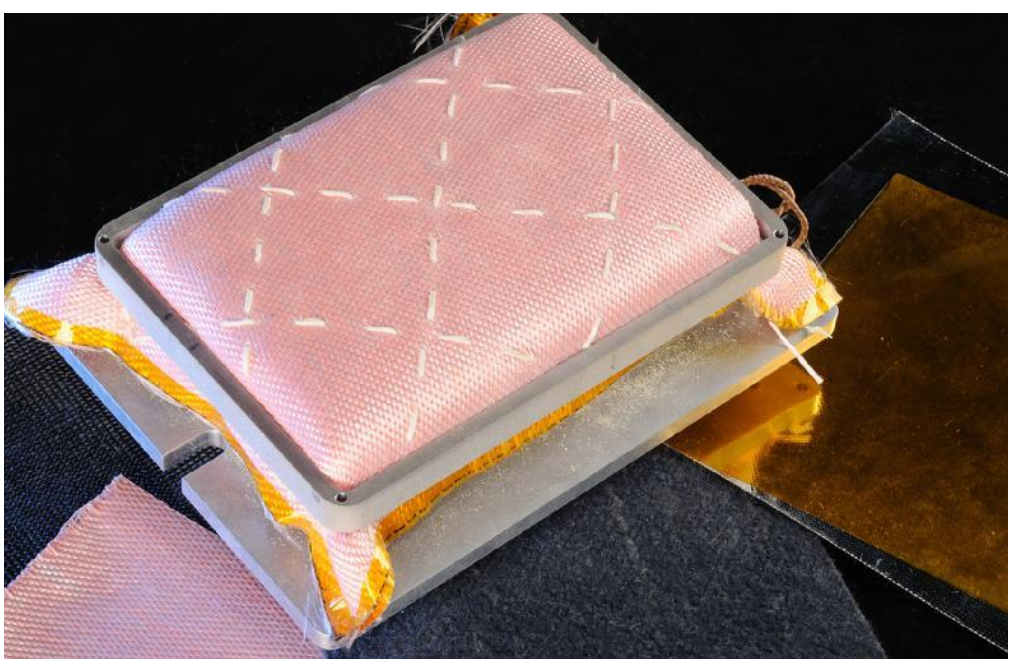

Figure 4. TPS test article tensioned across rigid tile, exhibiting a flat middle surface and parabolic edge curvature ${ }^{1}$.

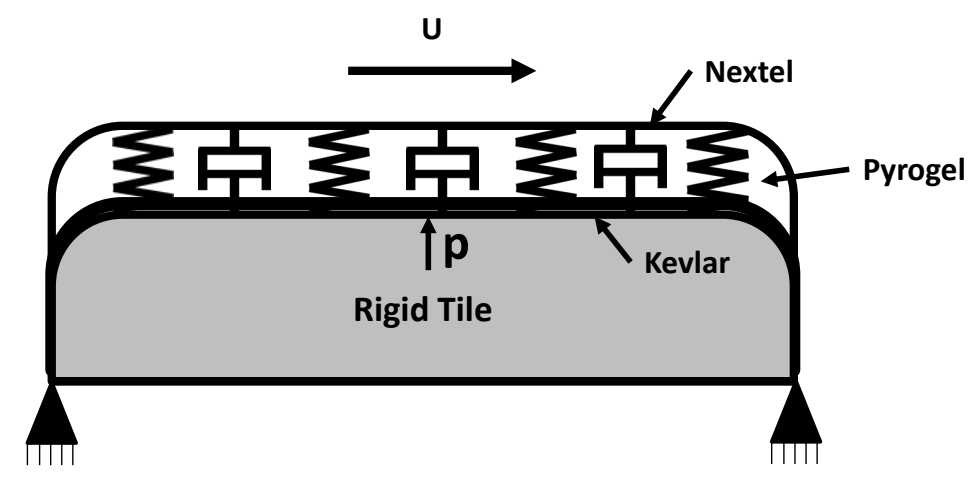

Figure 5. Curved TPS model due to imposed pressure from the rigid tile.

\section{Governing Equations}

A similar nonlinear stress formulation from the previous curvature model is used here, but with several key modifications. Again, these modifications apply to a single plate, but the equations for full multi-layer TPS can be easily derived following the procedure for the flat plate model in section A. In this case, the rise height of the initial curvature function is set to zero, such that the condition of a flat plate is attained. The curvature is imposed via a generalized force, which is calculated in the following manner. The desired curvature functions (in the $\mathrm{x}$ direction only) are:

$$
\begin{array}{ccc}
z_{1}=\frac{H^{b}}{x_{1}^{2}}\left[x_{1}^{2}-\left(\frac{x}{a}-x_{1}\right)^{2}\right] & x=\left[0, x_{1}\right] \\
z_{2}=H^{b} & x=\left[x_{1}, x_{2}\right] \\
z_{3}=\frac{H^{b}}{\left(1-x_{2}\right)^{2}}\left[\left(1-x_{2}\right)^{2}-\left(\frac{x}{a}-x_{2}\right)^{2}\right] & x=\left[x_{2}, a\right]
\end{array}
$$


Where $x_{1}$ and $x_{2}$ are the positions along the plate length where the edge curvature begins and ends, respectively. Since measurements of the rigid base are not yet available to the authors, the region of curvature at the edges was estimated to be ten percent of the length of the plate (based on the presentation of the curvature in Figure 4). For the purposes of convenient numerical integration, Eq. (1.19) is expanded in a Fourier series of the following form

$$
z=\sum_{n} c_{n} \alpha_{n}(x, y)
$$

The Fourier coefficients $c_{n}$ are given in the appendix. Not that while only a single index is written, summation is in both $\mathrm{x}$ and $\mathrm{y}$ directions. The resulting surface from the Fourier series expansion is shown in Figure 6.

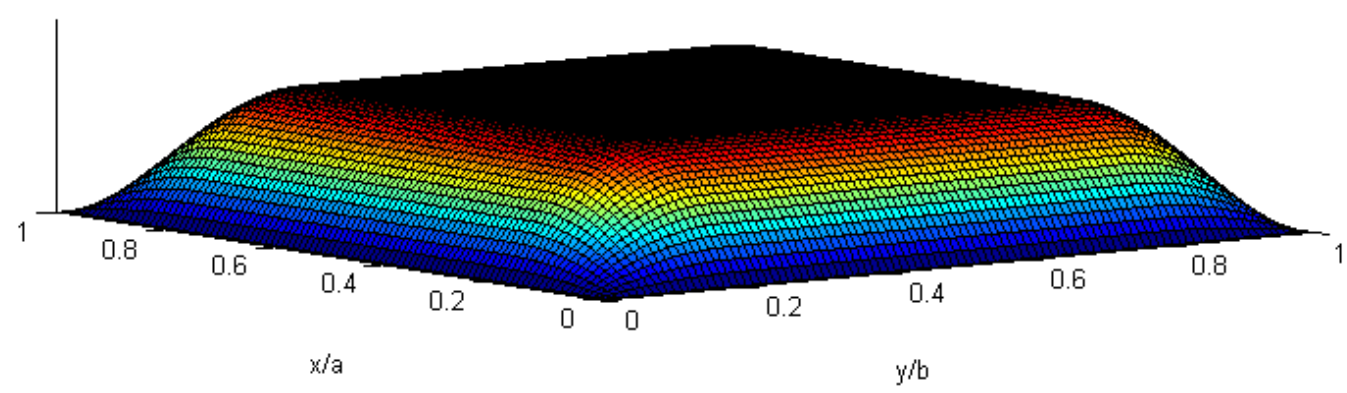

Figure 6. Fourier series representation of the rigid base geometry. Panel length and width have been non-dimensionalized.

The imposed pressure $p$ is calculated using the static form of Von Karman's large deflection equations:

$$
\begin{aligned}
& D \nabla^{4} z=\frac{\partial^{2} F_{z}}{\partial y^{2}} \frac{\partial^{2} z}{\partial x^{2}}+\frac{\partial^{2} F_{z}}{\partial x^{2}} \frac{\partial^{2} z}{\partial y^{2}}-2 \frac{\partial^{2} F_{z}}{\partial x \partial y} \frac{\partial^{2} z}{\partial x \partial y}+p \\
& \frac{\nabla^{4} F_{z}}{E h}=\frac{\partial^{2} w_{z}}{\partial x \partial y} \frac{\partial^{2} w_{z}}{\partial x \partial y}-\frac{\partial^{2} w_{z}}{\partial x^{2}} \frac{\partial^{2} w_{z}}{\partial y^{2}}
\end{aligned}
$$

With

$$
F_{z}(x, y)=\frac{\bar{N}_{y} x^{2}}{2}+\frac{\bar{N}_{x} y^{2}}{2}-\bar{N}_{x y} x y+\sum_{j} f_{j} \gamma_{j}(x, y)
$$

Numerical integration of (1.21) results in the generalized force term that can then be used in the dynamic equations of motion. The full expression is provided in the appendix. Again, stress-free and imposed-stress in-plane boundary conditions will be considered with simply supported out of plane conditions.

\section{Material Properties}

The physical properties for the three TPS materials are given in Table 1, while the nominal geometry and mechanical properties are given in Table 2. The materials studied in this paper include Nextel 400-BF20, Pyrogel 6650, and Aluminized Kapton Kevlar Laminate. 
Table 1. TPS material properties from material documentation and various literature sources ${ }^{5-7}$

\begin{tabular}{|c|c|c|c|}
\hline Material & 2x Pyrogel 6650 & 1x Kevlar & 2x Nextel 440* \\
\hline Thickness $-\mathrm{h}(\mathrm{m})$ & 0.00598 & 0.000127 & 0.001016 \\
\hline Density $-\rho\left(\mathrm{kg} / \mathrm{m}^{3}\right)$ & 112 & 1440 & 3050 \\
\hline Mass/area - $\mathrm{m}\left(\mathrm{kg} / \mathrm{m}^{2}\right)$ & 0.67 & 0.18 & 3.1 \\
\hline Young's (tensile) modulus - E $(\mathrm{Pa})$ & $3.80 \times 10^{6}$ & $1.13 \times 10^{11}$ & $1.90 \times 10^{11}$ \\
\hline Poisson ratio $-v$ & 0.20 & 0.36 & 0.36 \\
\hline Bending rigidity $-\mathrm{D}\left(\mathrm{Pa} \mathrm{m}{ }^{3}\right)$ & - & 0.022 & 2.39 \\
\hline Spring stiffness $-\mathrm{k}(\mathrm{Pa} / \mathrm{m})$ & $6.60 \times 10^{8}$ & - & - \\
\hline
\end{tabular}

Table 2. Nominal configuration geometric and structural properties

\begin{tabular}{|l|l|}
\hline Sample Length $-\mathrm{a}(\mathrm{m})$ & 0.1778 \\
\hline Sample Width $-\mathrm{b}(\mathrm{m})$ & 0.127 \\
\hline Nextel Damping Ratio $-\zeta_{\mathrm{N}}$ & 0 \\
\hline Kevlar Damping Ratio $-\zeta_{\mathrm{K}}$ & 0 \\
\hline In-plane loads (tension) $-\mathrm{N}_{\mathrm{x}}, \mathrm{N}_{\mathrm{Y}}(\mathrm{N} / \mathrm{m})$ & 0 \\
\hline
\end{tabular}

*Note: The bending rigidity for the Nextel was calculated assuming two sandwiched layers as in the layup configuration ${ }^{1}$; however, it was determined from preliminary experiments that the bending rigidity of two layers was not equal to twice that of one layer. In fact, the measurements for both cases were nearly the same. As a result, and for the purposes of determining the lower bound on flutter, the bending rigidity of one layer of Nextel was used in all calculations. Also, the two layers of Pyrogel are modeled as a single layer with twice the nominal thickness. Additional structural tests to measure the bending properties directly are currently in progress.

\section{Natural Frequency Results and Analysis}

The natural frequency results are presented in the following section for all three theoretical models. For the linear flat plate model, calculations were made using an eigenvalue solver, while the calculations for the two nonlinear models were made using a time-marching algorithm in MATLAB.

For each of the three structural models, various configurations of the TPS materials were investigated. The following specifications are used to reference to the different configurations: when a reference is made to "Nextel Alone" or "Kevlar Alone," the other TPS layers are not included in the calculation but all other properties of the structural model remain the same. This would apply if there is no knitting connecting any of the layers together in the test setup. When a reference is made to a material on a "Pyrogel foundation," the material rests on the Pyrogel layer which is itself resting on a rigid base. When all three layers of the TPS are included in the analysis, it will be referred to as the "3-layer TPS." These specifications will be used in the discussion of the flutter results as well.

\section{A. Linear Flat Plate Model}

The natural frequencies were calculated for several configurations of the linear flat plate model, including the Nextel alone, Kevlar alone, and 3-layer TPS, with and without in-plane loads. In addition, both simply-supported and clamped boundary conditions were considered. All of these results are presented in Table 3-5. 
Table 3. Natural Frequencies of various Nextel configurations in $\mathrm{rad} / \mathrm{s}$.

\begin{tabular}{|c|c|c|c|c|c|c|}
\hline Mode & $\begin{array}{c}\text { Nextel } \\
\text { Alone, } \\
\text { Nominal } \\
\text { (BC: SS) }\end{array}$ & $\begin{array}{c}\text { Nextel Alone } \\
\mathbf{N}_{\mathbf{x}}=\mathbf{N}_{\mathbf{y}}= \\
\mathbf{1 0 0 0} \mathbf{N} / \mathbf{m} \\
\text { (BC: } \mathbf{S S} \text { ) }\end{array}$ & $\begin{array}{c}\text { Nextel on } \\
\text { Pyrogel } \\
\text { Foundation } \\
\text { (BC: } \mathbf{S S} \text { ) }\end{array}$ & $\begin{array}{c}\text { Nextel Alone, } \\
\text { Nominal } \\
\text { (BC: Clamped) }\end{array}$ & $\begin{array}{c}\text { Nextel Alone } \\
\mathbf{N}_{\mathbf{x}}=\mathbf{N}_{\mathbf{y}}= \\
\mathbf{1 0 0 0} \mathbf{N} / \mathbf{m} \\
\text { (BC: } \mathbf{C l a m p e d )}\end{array}$ & $\begin{array}{c}\text { Nextel on } \\
\text { Pyrogel } \\
\text { Foundation } \\
\text { (BC: Clamped) }\end{array}$ \\
\hline 1 & 1405 & 1694 & 25353 & 2641 & 2845 & 25451 \\
\hline 2 & 2828 & 3132 & 25471 & 4287 & 4531 & 25674 \\
\hline 3 & 5201 & 5511 & 25842 & 7019 & 7284 & 26269 \\
\hline 4 & 8523 & 8837 & 26710 & 10767 & 11043 & 27508 \\
\hline 5 & 12795 & 13110 & 28363 & 15491 & 15774 & 29677 \\
\hline 6 & 18015 & 18331 & 31069 & 21175 & 21463 & 33002 \\
\hline 7 & 24184 & 24502 & 35009 & 27849 & 28141 & 37635 \\
\hline 8 & 31303 & 31621 & 40257 & 35460 & 35755 & 43569 \\
\hline 9 & 39371 & 39689 & 46806 & 41580 & 41859 & 48679 \\
\hline 10 & 48388 & 48706 & 54609 & 48227 & 48580 & 54467 \\
\hline
\end{tabular}

Table 4. Natural Frequencies of various Kevlar configurations in rad/s

\begin{tabular}{|c|c|c|c|c|c|c|}
\hline Mode & $\begin{array}{c}\text { Kevlar } \\
\text { Alone, } \\
\text { Nominal } \\
\text { (BC: SS) }\end{array}$ & $\begin{array}{c}\text { Kevlar } \\
\text { Alone } \\
\mathbf{N}_{\mathbf{x}}=\mathbf{N}_{\mathbf{y}}= \\
\mathbf{1 0 0 0} \mathbf{N} / \mathbf{m} \\
\text { (BC: } \mathbf{S S} \text { ) }\end{array}$ & $\begin{array}{c}\text { Kevlar on } \\
\text { Pyrogel } \\
\text { Foundation } \\
\text { (BC: SS) }\end{array}$ & $\begin{array}{c}\text { Kevlar Alone, } \\
\text { Nominal } \\
\text { (BC: Clamped) }\end{array}$ & $\begin{array}{c}\text { Kevlar Alone } \\
\mathbf{N}_{\mathbf{x}}=\mathbf{N}_{\mathbf{y}}= \\
\mathbf{1 0 0 0} \mathbf{\text { Nm }} \\
\text { (BC: } \text { Clamped) }\end{array}$ & $\begin{array}{c}\text { Kevlar on } \\
\text { Pyrogel } \\
\text { Foundation } \\
\text { (BC: Clamped) }\end{array}$ \\
\hline 1 & 323 & 2289 & 60554 & 607 & 2602 & 60556 \\
\hline 2 & 651 & 3280 & 60557 & 986 & 3647 & 60561 \\
\hline 3 & 1196 & 4521 & 60565 & 1614 & 4922 & 60575 \\
\hline 4 & 1960 & 5916 & 60585 & 2476 & 6366 & 60604 \\
\hline 5 & 2943 & 7444 & 60624 & 3563 & 7958 & 60658 \\
\hline 6 & 4143 & 9111 & 60695 & 4870 & 9698 & 60749 \\
\hline 7 & 5562 & 10924 & 60808 & 6405 & 11599 & 60891 \\
\hline 8 & 7199 & 12893 & 60979 & 8155 & 13663 & 61100 \\
\hline 9 & 9055 & 15029 & 61226 & 9563 & 14982 & 61303 \\
\hline 10 & 11129 & 17340 & 61567 & 11092 & 17846 & 61560 \\
\hline
\end{tabular}

Table 5. Natural frequencies of the 3-Layer TPS in rad/s.

\begin{tabular}{|c|c|c|c|c|}
\hline Mode & $\begin{array}{c}\text { 3-Layer TPS, Nominal } \\
\text { (BC: SS) }\end{array}$ & $\begin{array}{c}\text { 3-Layer TPS, } \\
\mathbf{N}_{\mathbf{x}}=\mathbf{N}_{\mathbf{y}}= \\
\mathbf{1 0 0 0} \mathbf{~ N} / \mathbf{m} \\
(\text { BC: } \mathbf{S S} \text { ) }\end{array}$ & $\begin{array}{c}\text { 3-Layer TPS, } \\
\text { Nominal } \\
\text { (BC: Clamped) }\end{array}$ & $\begin{array}{c}\text { 3-Layer TPS, } \\
\mathbf{N}_{\mathbf{x}}=\mathbf{N}_{\mathbf{y}}= \\
\mathbf{1 0 0 0} \mathbf{~} / \mathbf{m} \\
\text { (BC: } \mathbf{C l a m p e d})\end{array}$ \\
\hline 1 & 726 & 1001 & 1364 & 1566 \\
\hline 2 & 1461 & 1758 & 2211 & 2456 \\
\hline 3 & 2680 & 2992 & 3608 & 3877 \\
\hline 4 & 4370 & 4691 & 5493 & 5780 \\
\hline 5 & 6492 & 6824 & 7792 & 8097 \\
\hline 6 & 50781 & 50799 & 50812 & 50835 \\
\hline 7 & 50818 & 50856 & 50882 & 50927 \\
\hline 8 & 50936 & 51005 & 51074 & 51152 \\
\hline 9 & 51219 & 51331 & 51488 & 51611 \\
\hline 10 & 51787 & 51952 & 52264 & 52441 \\
\hline
\end{tabular}

11

American Institute of Aeronautics and Astronautics 
The natural frequencies for the various linear flat plate configurations have several important properties. First, the effect of applying tension at the boundaries of $1000 \mathrm{~N} / \mathrm{m}$ had surprisingly little effect on the Nextel alone, while the natural frequencies of the Kevlar increased significantly. This same loading had a minimal effect on the 3-layer system, which suggests that the stiffer Nextel governs the TPS dynamics and should also govern the flutter behavior. However, the natural frequencies of the 3-layer TPS are quite different than the natural frequencies of the Nextel alone. The first five modes are lower than the corresponding modes of the Nextel, which is due to the added inertia of the Pyrogel and Kevlar. The next five modes are significantly higher than those of the Nextel alone, but this is because five modes were used for both the Nextel and Kevlar in the 3-layer TPS eigenvalue calculation. Since the Nextel is orders of magnitude more rigid than the Kevlar, the Kevlar behaves as if there is a Pyrogel foundation, resulting in the higher natural frequencies. Finally, the clamped boundary conditions tend to increase the natural frequencies by approximately a factor of 2 with respect to simply supported boundaries for the lower modes. This effect diminishes when the Pyrogel foundation is added to the Nextel and Kevlar because the Pyrogel spring stiffness is quite large compared to the plate bending stiffness.

\section{B. Parabolic Curvature Model}

Although this model includes the Nextel, Pyrogel, and Kevlar, natural frequencies of the Nextel alone were also computed. Figure 7 illustrates the effect of streamwise and spanwise curvature on the natural frequencies of the Nextel alone. When $\mathrm{H} / \mathrm{h}$ is zero, there is no curvature, and a flat plate is attained. The natural frequencies for this case are nearly the same as those calculated in the linear flat plate analysis. Two different computational/numerical methods were used for these calculations, so obtaining nearly the same results provides some degree of verification to both methods.

When the Nextel has curvature in both directions, all of the natural frequencies increase as $\mathrm{H} / \mathrm{h}$ increases $(\mathrm{H}$ and $\mathrm{h}$ are the rise height and plate thickness, respectively). With streamwise curvature only, shown in Figure 8, the natural frequencies intersect at various locations corresponding to different values of $\mathrm{H} / \mathrm{h}$. At these intersection points, one might expect the plate to be more susceptible to flutter since eigenvalue coalescence has occurred. ${ }^{4}$ It will be shown later that in this case, the flutter boundary is usually lower than for a flat plate.

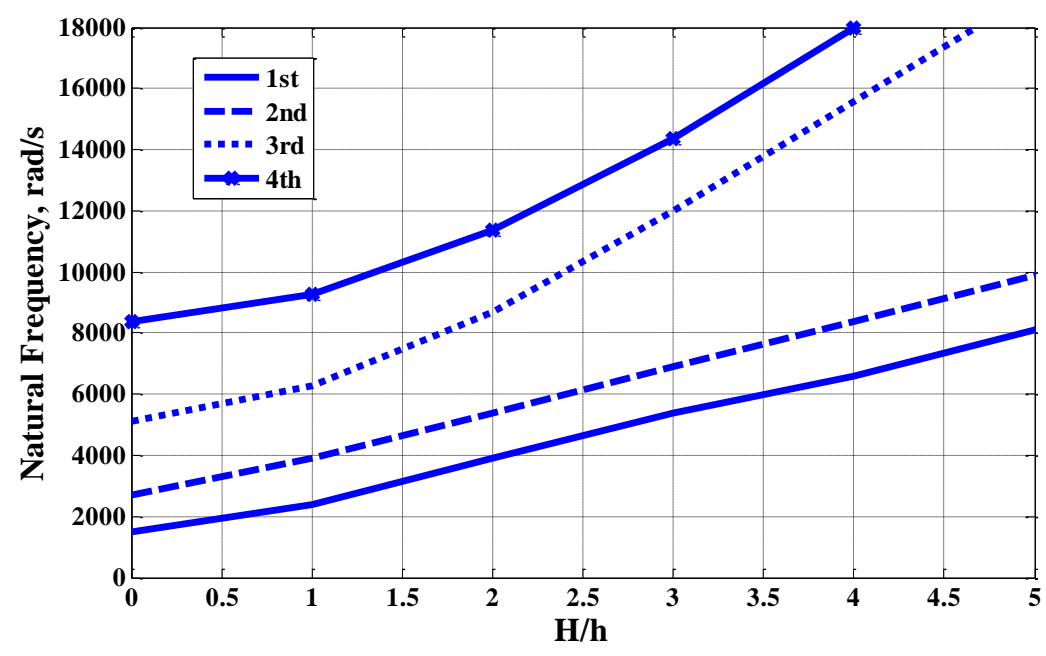

Figure 7. First four natural frequencies of the Nextel alone as a function of $\mathrm{H} / \mathrm{h}$, with both streamwise and spanwise curvature. 


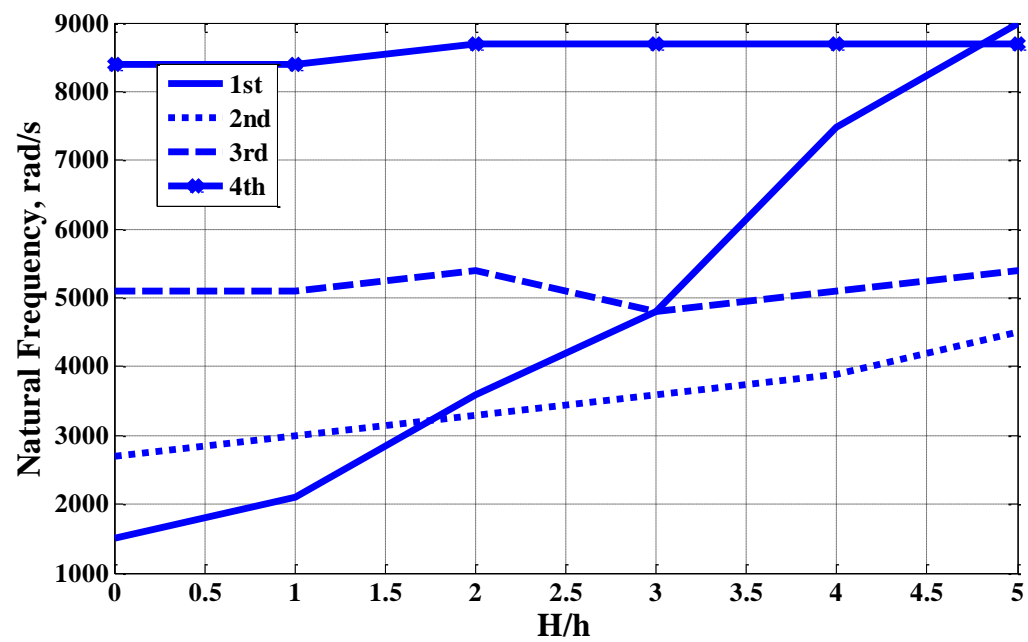

Figure 8. First four natural frequencies of the Nextel alone as a function of $\mathrm{H} / \mathrm{h}$, with streamwise curvature only.

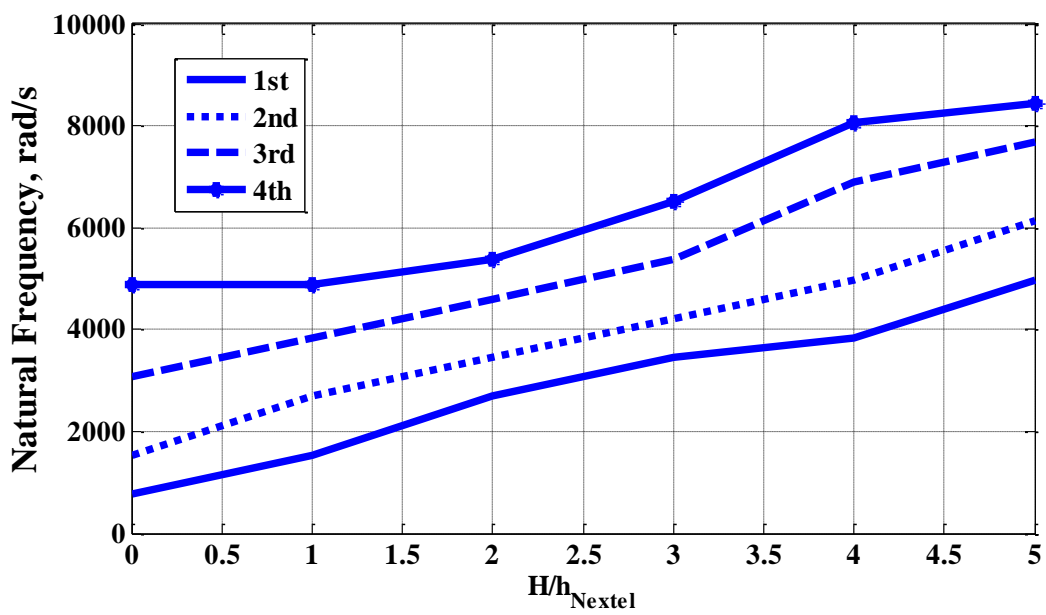

Figure 10. First four natural frequencies of the 3-layer TPS as a function of $\mathrm{H} / \mathrm{h}$, with both streamwise and spanwise curvature.

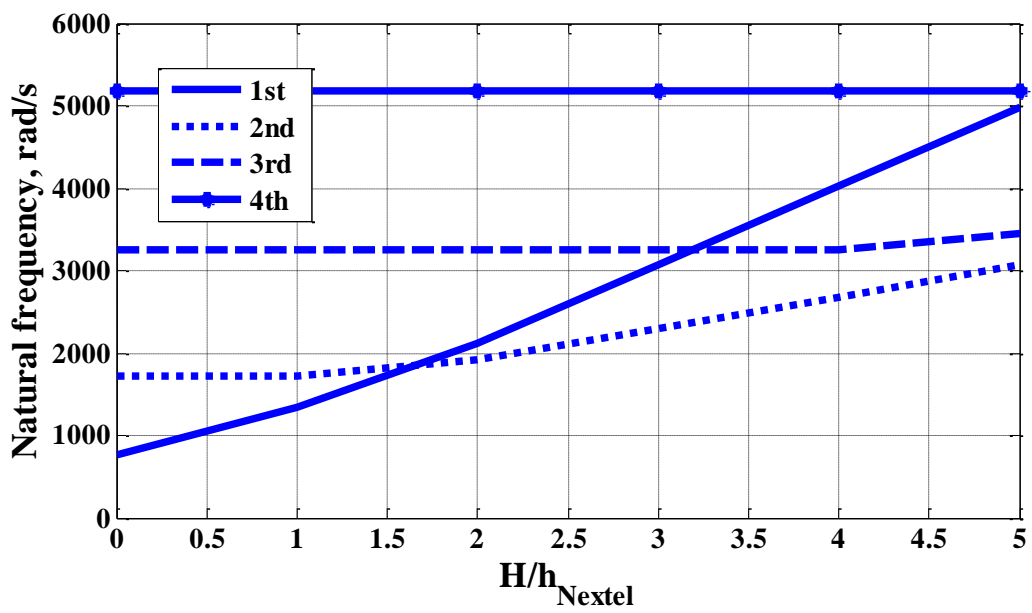

Figure 9. First four natural frequencies of the 3-layer TPS as a function of $\mathrm{H} / \mathrm{h}$, with streamwise curvature only. 
The natural frequencies for the 3-layer TPS are given in Figure 9 and Figure 10. The general trend of these natural frequencies is similar to those for the Nextel alone, while the magnitudes of the frequencies are lower. In the case of streamwise curvature only, the points of intersection between the modes are nearly identical to the Nextel. Again, this would indicate that the flutter behavior of the Nextel is similar to that of the 3-layer TPS, and the results in the next section indicate that this is generally the case.

\section{Imposed Pressure Model}

The natural frequencies for this model are the same as the parabolic curvature model with $\mathrm{H} / \mathrm{h}$ equal to zero, corresponding to a flat plate. This is because the curvature is induced by an external forcing, rather than the geometric configuration of the material.

\section{Flutter Results and Analysis}

The flutter results that follow will be presented in terms of dimensional parameters for easier comparison to the supersonic/hypersonic wind tunnel tests. The Mach number will be fixed to a value of 5, and the flow density will describe the flutter point. Computations have also been done with the flow density fixed and the Mach number varied, but these results will be omitted for the sake of brevity.

\section{A. Linear Flat Plate Model}

\section{Flutter of the Nextel in various configurations}

Table 6 includes the flutter densities of Nextel with in-plane loads, structural damping, and with a Pyrogel foundation. The results indicate that low-mode flutter is possible at $\mathrm{M}=5$ for a flow density significantly below that of air at sea level $\left(\rho_{\mathrm{sl}}\left(20^{\circ} \mathrm{C}\right)=1.2 \mathrm{~kg} / \mathrm{m}^{3}\right)$, for both simply supported and clamped boundaries on the Nextel alone. Addition of a relatively stiff Pyrogel foundation increases both the natural and flutter frequencies dramatically, which is likely non-physical due to the fact that the Nextel is not bonded at every point to the Pyrogel and the Kevlar motion is neglected. It is also seen that the addition of structural damping for the Nextel alone has little effect on the flutter results. This is consistent with the conclusions of Dowell. ${ }^{3}$ Structural damping is much more significant when a Pyrogel spring foundation is added since the damping generalized force term is proportional to the fundamental natural frequency $(\sqrt{k / m})$.

Table 6. Flutter results for the Nextel in various configurations, with $M=5$.

\begin{tabular}{|c|c|c|c|}
\hline Configuration & $\begin{array}{c}\text { Flutter Frequency } \\
\omega_{\mathrm{f}}(\mathrm{rad} / \mathrm{s})\end{array}$ & $\begin{array}{c}\text { Flutter Density } \\
\rho_{\mathrm{f}}\left(\mathrm{kg} / \mathrm{m}^{3}\right)\end{array}$ & $\begin{array}{l}\text { Flutter } \\
\text { Modes }\end{array}$ \\
\hline Nextel Alone, Nominal (BC: SS) & 2578 & 0.51 & 1,2 \\
\hline $\begin{array}{c}\text { Nextel Alone }(\mathrm{BC}: \mathrm{SS}) \\
\mathrm{Nx}=\mathrm{Ny}=1000 \mathrm{~N} / \mathrm{m}\end{array}$ & 2924 & 0.61 & 1,2 \\
\hline $\begin{array}{c}\text { Nextel Alone (BC: SS) } \\
\zeta_{N}=0.05\end{array}$ & 2606 & 0.53 & 1,2 \\
\hline $\begin{array}{c}\text { Nextel on Pyrogel Foundation } \\
\text { (BC: SS) }\end{array}$ & 25679 & 1.41 & 1,2 \\
\hline $\begin{array}{c}\text { Nextel on Pyrogel Foundation } \\
\text { (BC: SS) } \\
\zeta_{\mathrm{N}}=0.05\end{array}$ & 27222 & 5.72 & 1,2 \\
\hline Nextel Alone, Nominal (BC: Clamped) & 3954 & 0.76 & 1,2 \\
\hline $\begin{array}{l}\text { Nextel Alone (BC: Clamped) } \\
\mathrm{Nx}=\mathrm{Ny}=1000 \mathrm{~N} / \mathrm{m}\end{array}$ & 4224 & 0.85 & 1,2 \\
\hline $\begin{array}{l}\text { Nextel Alone (BC: Clamped) } \\
\zeta_{\mathrm{N}}=0.05\end{array}$ & 3996 & 0.8 & 1,2 \\
\hline $\begin{array}{l}\text { Nextel on Pyrogel Foundation } \\
\text { (BC: Clamped) }\end{array}$ & 25856 & 1.67 & 1,2 \\
\hline $\begin{array}{c}\text { Nextel on Pyrogel Foundation } \\
\text { BC: Clamped } \\
\zeta_{\mathrm{N}}=0.05\end{array}$ & 27443 & 6.06 & 1,2 \\
\hline
\end{tabular}

American Institute of Aeronautics and Astronautics 
Note that simply supported boundary conditions require that there be no deflection or bending moment at the edges. Clamped boundary conditions require that the plate slope must also vanish at the edges in addition to the deflection. Since this condition is difficult to enforce in an experimental setup, it is likely that the TPS test article is closer to simply supported than clamped. In any case, the Nextel alone with simply supported boundary conditions has the lowest flutter density of all the Nextel configurations.

\section{Flutter of the 3-layer TPS}

The flutter results in Table 7 are for the 3-layer TPS with various structural properties. Note that the boundary conditions indicated apply to the Nextel, Pyrogel, and Kevlar layers.

\begin{tabular}{|c|c|c|c|}
\hline $\begin{array}{c}\text { Configuration of the } 3 \text { - } \\
\text { layer TPS }\end{array}$ & $\begin{array}{c}\text { Flutter Frequency } \omega_{\mathrm{f}} \\
(\mathrm{rad} / \mathrm{s})\end{array}$ & $\begin{array}{c}\text { Flutter } \\
\text { Density } \rho_{\mathrm{f}} \\
\left(\mathrm{kg} / \mathrm{m}^{3}\right)\end{array}$ & $\begin{array}{l}\text { Flutter } \\
\text { Modes }\end{array}$ \\
\hline Nominal (BC: SS) & 1326 & 0.52 & 1,2 \\
\hline $\begin{array}{l}\text { Pyrogel Mass }=0 \\
\text { (BC: SS) }\end{array}$ & 2340 & 0.52 & 1,2 \\
\hline $\begin{array}{c}\mathrm{N}_{\mathrm{x}}=\mathrm{N}_{\mathrm{y}}=1000 \mathrm{~N} / \mathrm{m} \\
\text { (BC: } \mathrm{SS} \text { ) }\end{array}$ & 1662 & 0.72 & 1,2 \\
\hline $\begin{array}{c}\zeta_{\mathrm{N}}=\zeta_{, K}=0.05 \\
(\text { BC: } \mathrm{SS})\end{array}$ & 1326 & 0.52 & 1,2 \\
\hline $\begin{array}{c}\zeta_{N}=\zeta_{, K}=0.1 \\
\text { BC: }: S S\end{array}$ & 1339 & 0.524 & 1,2 \\
\hline Nominal (BC: Clamped) & 2021 & 0.76 & 1,2 \\
\hline $\begin{array}{c}\text { Pyrogel Mass }=0 \\
(\text { BC: Clamped) }\end{array}$ & 3579 & 0.77 & 1,2 \\
\hline $\begin{array}{l}\mathrm{N}_{\mathrm{x}}=\mathrm{N}_{\mathrm{y}}=1000 \mathrm{~N} / \mathrm{m} \\
\text { (BC: Clamped) }\end{array}$ & 2283 & 0.93 & 1,2 \\
\hline $\begin{array}{c}\zeta_{N}=\zeta_{, K}=0.05 \\
(B C: \text { Clamped) }\end{array}$ & 2026 & 0.77 & 1,2 \\
\hline $\begin{array}{c}\zeta_{\mathrm{N}}=\zeta_{\mathrm{K}}=0.1 \\
(\mathrm{BC}: \text { Clamped })\end{array}$ & 2037 & 0.79 & 1,2 \\
\hline
\end{tabular}

Table 7: Flutter results for 3-layer TPS in various configurations. $M=5$.

It is observed that low-mode flutter occurs for all configurations of the 3-layer TPS. For any given configuration above, the clamped cases result in higher flutter frequencies and flutter densities than the equivalent simply supported cases. When the Pyrogel mass is neglected, the flutter frequency increases, which is expected since the effective inertia of the system is lower. Tensioning the TPS by a load of $1000 \mathrm{~N} / \mathrm{m}$ only increases the flutter density by a factor of 1.2 to 1.4 depending on the boundary conditions. Since an in-plane load of $1000 \mathrm{~N} / \mathrm{m}$ is quite large compared to what would be realistically applied, it is unlikely that tensioning will have a major effect on the flutter behavior in the wind-tunnel experiments. The effect of structural damping, even with a damping ratio as high as 0.1, also has little effect on the flutter densities.

The dynamics of the problem may be further explored by comparing the results from the 3-layer TPS to those of the Nextel alone. Consider the simply supported case only. The flutter densities are virtually the same for the 3-layer TPS and Nextel alone, which further indicates that the Nextel stiffness is likely governing the flutter behavior. When the Pyrogel mass is neglected, the flutter frequency of the 3-layer TPS approaches the flutter frequency of the Nextel alone, but is much lower when the Pyrogel mass is included since it adds inertia to the system. The effect of tensioning the 3-layer TPS by $1000 \mathrm{~N} / \mathrm{m}$ has a greater effect than for the Nextel alone, which is likely due to the added stiffness of the bottom Kevlar layer that is introduced upon tensioning. Finally, the results in Table 7 illustrate the significance of allowing deflection of the Kevlar. The relatively large stiffness of the Pyrogel is included, yet the high frequencies and large flutter densities seen with the Nextel on Pyrogel foundation are no longer present. The freedom of the Kevlar now allows the Nextel to deflect without the Pyrogel restricting its motion. This is confirmed by relative motion seen in the flutter mode shapes computed with nonlinear theory in the next section. 


\section{B. Parabolic Curvature Model}

For this model, only simply-supported boundary conditions are considered, since the linear analysis indicated these conditions resulted in the lowest flutter density, and only the lower bound on flutter is desired. For the same reasons, structural damping and applied in-plane loads have been neglected in these calculations. Note that calculations with in-plane loading of $1000 \mathrm{~N} / \mathrm{m}$ were performed, and the flutter density was unaffected at moderate to large $\mathrm{H} / \mathrm{h}$ values.

\section{Flutter of the Nextel alone}

Before considering the effect of initial parabolic curvature on the flutter behavior of the Nextel alone, it may be of interest to compare the three dimensional nonlinear flutter results with $\mathrm{H} / \mathrm{h}=0$ to the two-dimensional linear flat plate results computed earlier. Figure 11 shows that the peak in the flutter frequency spectrum of the nonlinear case is nearly aligned with linear flutter frequency. In addition, the flutter densities are the same, with $\rho_{\mathrm{f}} \sim 0.5$ at $\mathrm{M}=5$. This consistency adds validity to the numerical methods and computational implementations for both models, though the possibility of a systematic error is not excluded.

An example of the Nextel response just above the flutter boundary is shown in Figure $12 \mathrm{for} H / \mathrm{h}=5$. These limit cycle oscillations (LCOs) occur due to the geometric nonlinearity of the plate structure, which prevents the response from growing to infinity. It is evident that the response is highly chaotic, with average deflection amplitude of about five times the plate thickness. This result is consistent with the analysis of Dowell ${ }^{4}$, who found the flutter amplitude of curved plates to be on the order of the rise height, rather than the thickness. Also note that the baseline about which oscillation takes place is shifted below the zero point on the y-axis, which may indicate that plate initially deforms under the aerodynamic load and then flutters about that deformed position.

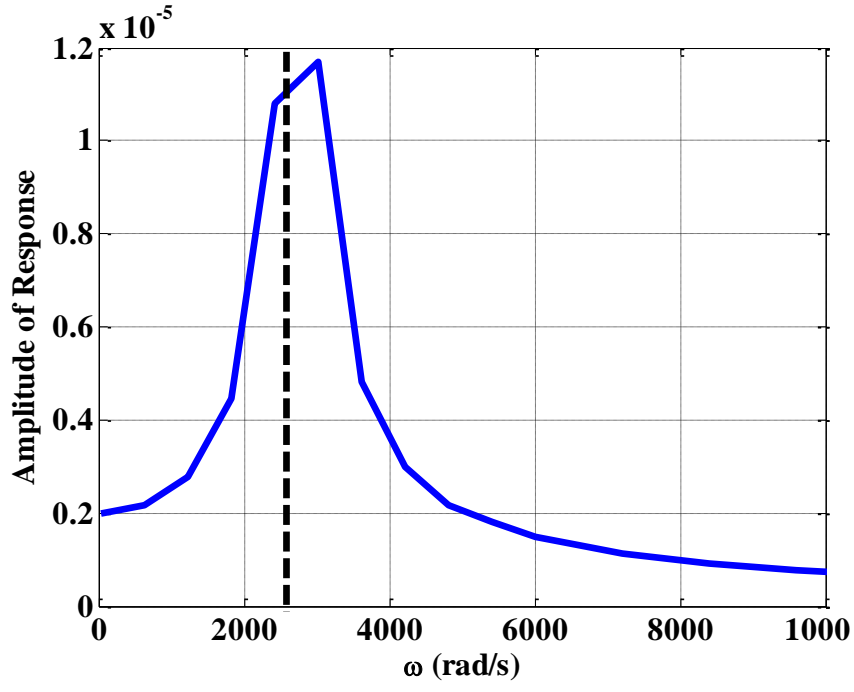

Figure 11. Flutter frequency spectrum for the Nextel alone with $H / h=0$. The dotted line indicates the two-dimensional linear flutter frequency.

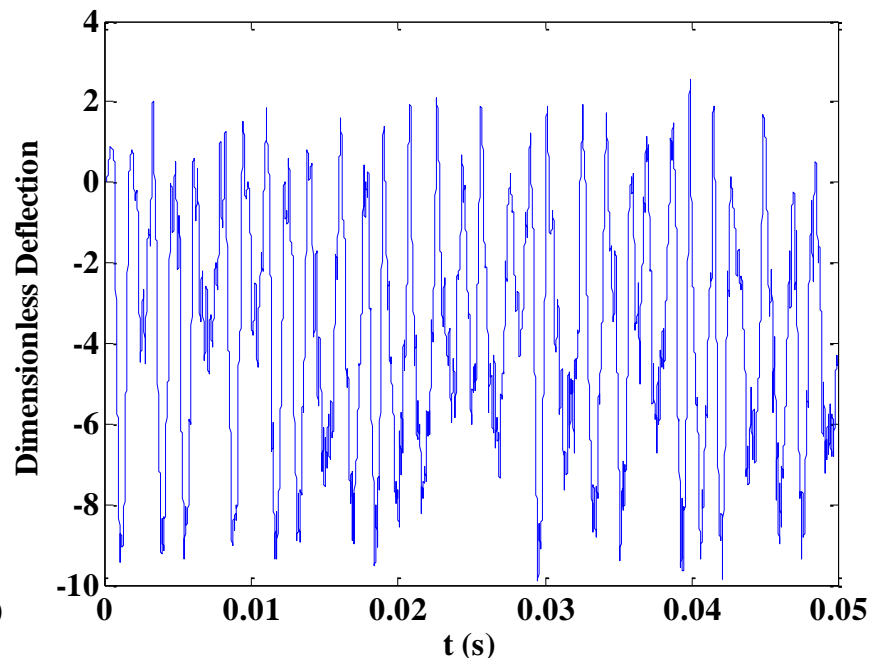

Figure 12. Nextel response (LCO) just above flutter boundary, with $\mathrm{H} / \mathrm{h}=\mathbf{5}$. Time history is sampled at a fixed location on the plate.

Table 8. Flutter densities for the Nextel alone at different values of $H / h$, with $M=5$.

\begin{tabular}{|c|c|c|c|c|}
\hline \multirow{2}{*}{$\mathbf{H} / \mathbf{h}$} & Flutter density $\boldsymbol{\rho}_{\mathbf{f}}\left(\mathbf{k g} / \mathbf{m}^{\mathbf{3}}\right)$ & \# of streamwise modes & Flutter density $\boldsymbol{\rho}_{\mathbf{f}}\left(\mathbf{k g} / \mathbf{m}^{\mathbf{3}}\right)$ & $\begin{array}{c}\text { \# of streamwise } \\
\text { modes }\end{array}$ \\
\cline { 2 - 5 } & & & 0.5 & 6 \\
\hline $\mathbf{0}$ & 0.5 & 6 & 0.4 & 6 \\
\hline $\mathbf{2}$ & 0.8 & 6 & 0.4 & 6 \\
\hline $\mathbf{3}$ & 1.1 & 8 & 0.4 & 8 \\
\hline $\mathbf{4}$ & 1.6 & 10 & 0.4 & 10 \\
\hline $\mathbf{5}$ & 1.6 & 12 & 0.4 & 12 \\
\hline
\end{tabular}


The flutter densities of the Nextel alone as a function of $\mathrm{H} / \mathrm{h}$ are given in Table 8 . With streamwise and spanwise curvature $(X \& Y)$, the flutter density increases as $\mathrm{H} / \mathrm{h}$ increases. When curvature is present only in the streamwise direction (X), the flutter density generally decreases. A similar qualitative result for streamwise curvature was found by Dowell ${ }^{4}$ with significantly different in-plane boundary conditions. The static aerodynamic loading due to the plate curvature (see Eqs. (1.16) and (A7)) is partially responsible for these effects, along with aerodynamic damping. Convergence is non-uniform and additional modes are required as $\mathrm{H} / \mathrm{h}$ increases. The number of streamwise modes needed for convergence at each value of $\mathrm{H} / \mathrm{h}$ is also given in Table 8 . For these calculations, one spanwise mode was retained. It was determined that in the case of only streamwise curvature, additional spanwise modes have no effect on the flutter density. With spanwise curvature, the flutter density increases slightly with additional odd spanwise modes, but remains unaffected for additional even spanwise modes. Since the effect is minimal and usually within the iteration tolerance for the flutter density, only one spanwise mode was retained. We would expect spanwise modes to be critical if the flow were three dimensional, but the effect is likely less pronounced here due to the simplified aerodynamic theory used in this analysis.

The flutter mode shape for the flat geometry is given in Figure 13, and the flutter mode shape for the parabolically curved geometry with $\mathrm{H} / \mathrm{h}=5$ is given in Figure 14. The flat geometry exhibits low mode flutter behavior, which was also seen in the linear results. The amplitude near the flutter boundary for this case is on the order of the thickness. The behavior of the Nextel with parabolic curvature is chaotic, and there is some influence from higher modes. Since the chaotic motion doesn't occur at a single frequency, there is no single flutter mode. The amplitude near the flutter boundary is on the order of the rise height, rather than the thickness. Analysis of the behavior in the full time series indicated snap-through buckling of the plate at non-periodic intervals. This behavior would likely be restricted in the wind tunnel layup configuration due to the presence of the rigid base.

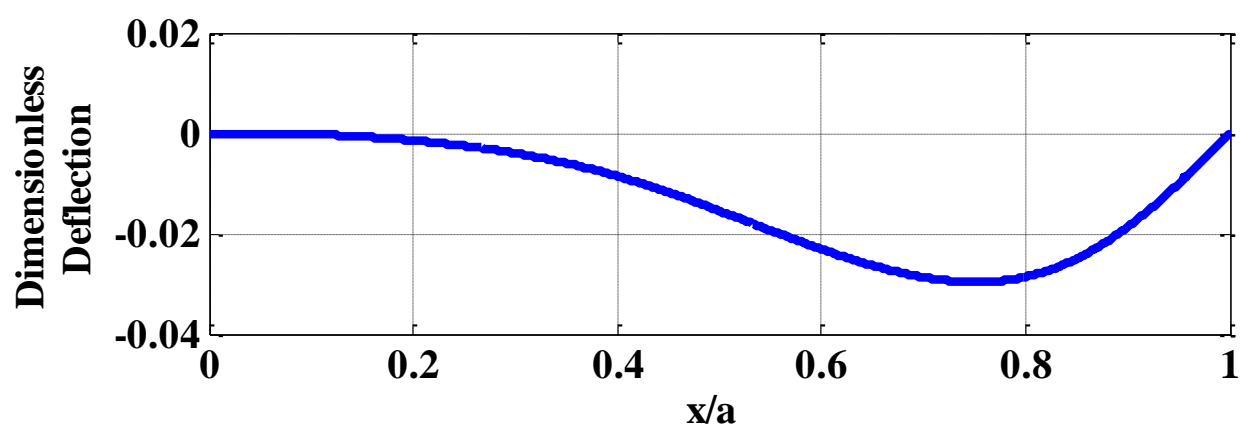

Figure 13. Flutter mode shape of the Nextel alone at an arbitrary time, with $H / h=0$. Deflection has been normalized by the Nextel thickness.

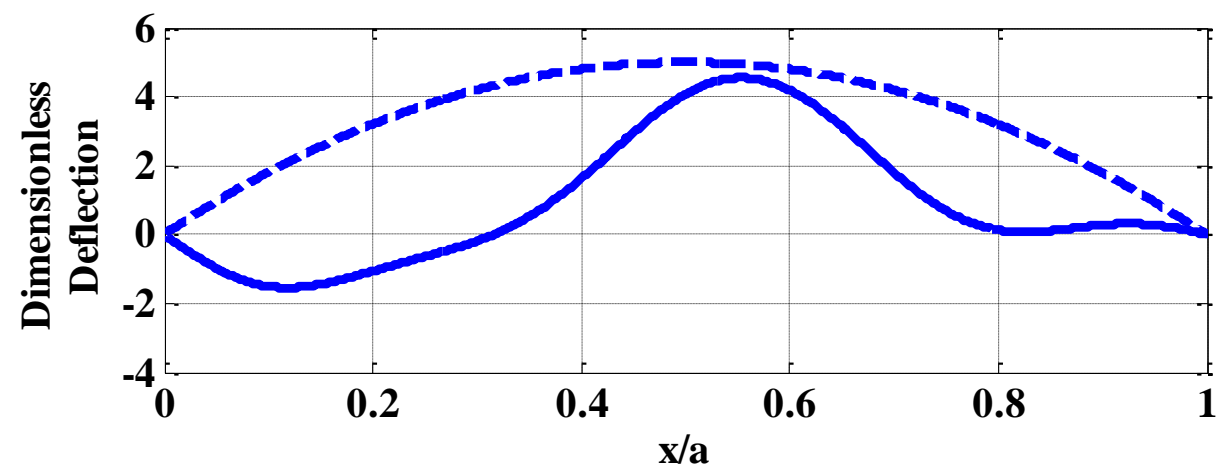

Figure 14. Flutter mode shape of the Nextel alone at an arbitrary time, with $H / h=5$. The dotted line indicates the initial parabolic shape. Deflection has been normalized by the Nextel thickness. 


\section{Flutter of the 3-layer TPS}

The flutter results for the 3-layer TPS are given in Table 9. The flutter density at $\mathrm{H} / \mathrm{h}=0$ is slightly larger than the density computed in the linear flat plate analysis, but by less than six percent. The flutter densities at all other values of $\mathrm{H} / \mathrm{h}$ are slightly less than those of the Nextel alone. The number of streamwise modes needed for convergence at large $\mathrm{H} / \mathrm{h}$ is also less than that of the Nextel. The limit cycle behavior is also chaotic for this system, similar to the behavior observed in Figure 12. Nextel response (LCO) just above flutter boundary, with $\mathrm{H} / \mathrm{h}=5$. Time history is sampled at a fixed location on the plate.Figure 12, but not shown here for brevity.

Table 9. Flutter densities for the 3-layer TPS at different values of $H / h$, with $M=5$.

\begin{tabular}{|c|c|c|c|c|}
\hline & \multicolumn{2}{|c|}{ Curvature in X \& Y } & \multicolumn{2}{c|}{ Curvature in X only } \\
\cline { 2 - 5 } & & & Flutter density $\boldsymbol{\rho}_{\mathbf{f}}$ \\
$\mathbf{H} / \mathbf{h}$ & Flutter density $\mathbf{\rho}_{\mathbf{f}}\left(\mathbf{k g} / \mathbf{m}^{\mathbf{3}}\right)$ & \# of streamwise modes & $\begin{array}{c}\text { \# of streamwise } \\
\text { modes }\end{array}$ \\
\hline $\mathbf{0}$ & & & 0.5 & 4 \\
\hline $\mathbf{1}$ & 0.5 & 4 & 0.4 & 4 \\
\hline $\mathbf{2}$ & 0.8 & 6 & 0.4 & 6 \\
\hline $\mathbf{3}$ & 1.0 & 6 & 0.3 & 8 \\
\hline $\mathbf{4}$ & 1.2 & 8 & 0.3 & 10 \\
\hline $\mathbf{5}$ & 1.2 & 10 & 0.3 & \\
\hline
\end{tabular}

The flutter mode shape for $\mathrm{H} / \mathrm{h}=0$ is given in Figure 15. Similar to the Nextel alone, the flat geometry of the 3layer TPS exhibits low mode flutter behavior, which was also indicated earlier by linear analysis. The high stiffness of the Pyrogel is evident because there is very little change in its nominal thickness during deflection. The free Kevlar base layer and Nextel generally deflect in unison along the panel length. Note that if the Pyrogel stiffness is decreased to well below its nominal value, it can be compressed easily and the Nextel and Kevlar are no longer strongly coupled.

Unlike the flat geometry, chaotic, large amplitude flutter is observed for the parabolic geometry in Figure 16. Snap-through behavior was also observed in the time series for this case.

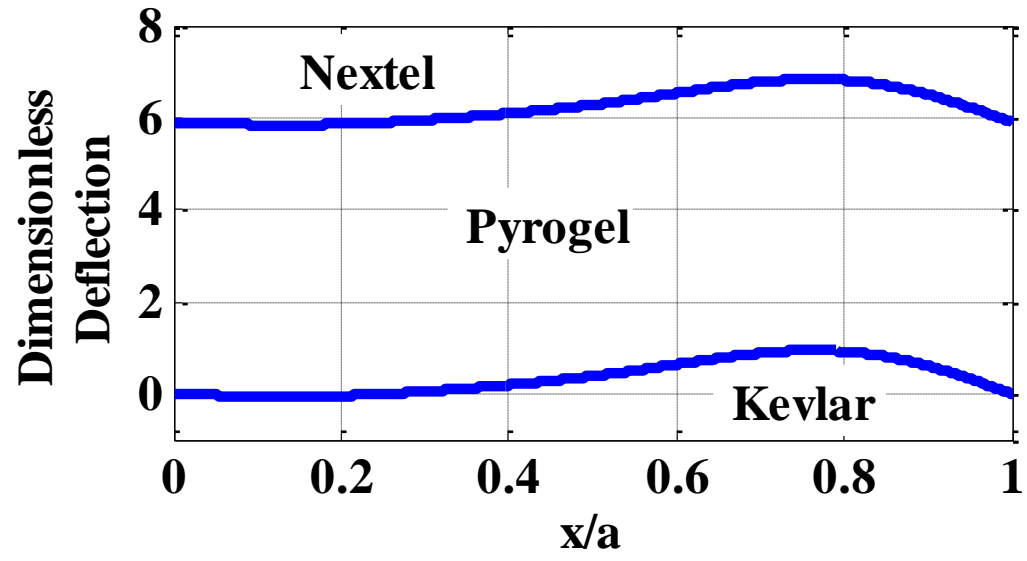

Figure 15. Flutter mode shape of the 3-layer TPS at an arbitrary time, with $\mathrm{H} / \mathrm{h}=\mathbf{0}$. Deflection has been normalized by the Nextel thickness. 


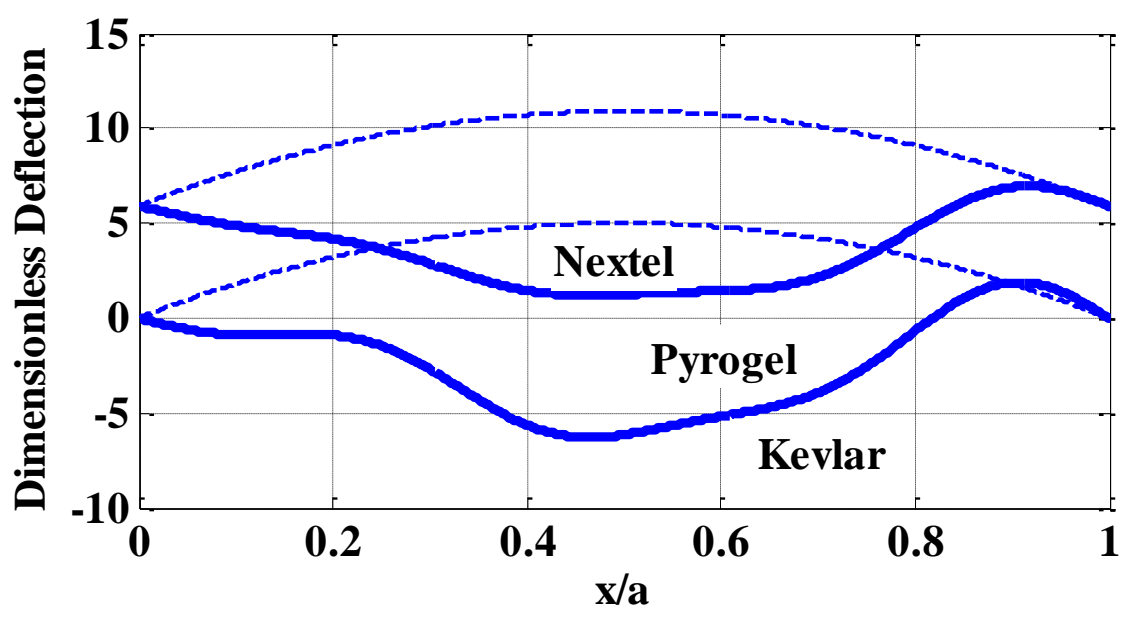

Figure 16. Flutter mode shape of the 3-layer TPS at an arbitrary time, with $H / h=5$. Deflection has been normalized by the Nextel thickness.

\section{Initial Conditions}

The simulations for both the Nextel alone and 3-layer TPS do not require specification of initial conditions when $\mathrm{H} / \mathrm{h}$ is greater than zero, due to the static pressure term in the aerodynamic forcing that provides a small initial deformation. However, these models respond quite differently when additional initial conditions are imposed. For example, when an initial displacement on the order of the thickness is imposed on the 3-layer TPS with $\mathrm{H} / \mathrm{h}=5$, the flutter density drops by over fifty percent and the limit cycle behavior is initially less chaotic. Since the wind-tunnel test article is initially undeformed beyond the initial curvature, no additional initial conditions were specified for the results previously presented.

\section{Imposed Pressure Model}

As in the previous model, only simply-supported, stress-free boundary conditions are considered. While there is edge curvature present in both the streamwise and spanwise directions, it was determined that resolution of the spanwise shape was unnecessary for the flutter calculations, though this may not be the case for other types of calculations. As a result, only the first spanwise mode was retained. Flutter was computed for both the Nextel alone and the 3-layer TPS.

\section{Flutter of the Nextel alone}

The flutter densities for the Nextel alone are given in Table 10. The flutter density increases rapidly as $\mathrm{H} / \mathrm{h}$ increases, which illustrates the inhibiting effect of static pressure on flutter. This effect was also observed by Dowell ${ }^{8}$ for the case of a constant, uniformly distributed pressure on a plate. The number of streamwise modes required for convergence is also much larger than the parabolic curvature model at large $\mathrm{H} / \mathrm{h}$. This is because the additional stress from the imposed pressure results in coalescence flutter of higher natural modes, thus requiring these higher modes for convergence.

Table 10: Flutter densities for the Nextel alone at different values of $H / h$, with $M=5$.

\begin{tabular}{|c|c|c|c|c|}
\hline $\mathbf{H} / \mathbf{h}$ & $\begin{array}{c}\text { Flutter density } \boldsymbol{\rho}_{\mathbf{f}} \\
\left(\mathbf{k g} / \mathbf{m}^{\mathbf{3}}\right)\end{array}$ & \# of streamwise modes & $\begin{array}{c}\text { Peak in FFT } \\
(\mathbf{r a d} / \mathbf{s})\end{array}$ & $\begin{array}{c}\text { Approx. Flutter } \\
\text { Modes }\end{array}$ \\
\hline $\mathbf{0}$ & 0.5 & 6 & 2174 & 1,2 \\
\hline $\mathbf{1}$ & 1.9 & 6 & 6474 & 3,4 \\
\hline $\mathbf{2}$ & 4.0 & 8 & 10827 & 4,5 \\
\hline $\mathbf{3}$ & 6.6 & 12 & 20933 & 6,7 \\
\hline $\mathbf{4}$ & 9.0 & 14 & 26166 & 7,8 \\
\hline $\mathbf{5}$ & 11.0 & 18 & 48099 & 9,10 \\
\hline
\end{tabular}

American Institute of Aeronautics and Astronautics 
Limit cycle oscillations (LCOs) of the Nextel just above the flutter boundary are shown in Figure 19 for $\mathrm{H} / \mathrm{h}=5$. The period between $\mathrm{t}=0 \mathrm{~s}$ and $\mathrm{t}=0.005 \mathrm{~s}$ is the time required to impose the static pressure, after which the aerodynamic pressure is applied. Despite a chaotic initial transient, the LCO is generally periodic, with amplitude on the order of the Nextel thickness rather than the rise height. This is in contrast to the LCO of the Nextel with parabolic curvature in Figure 12, which is chaotic with large amplitude oscillations near the flutter boundary. The periodic behavior of this system allowed for a peak frequency to be extracted from the Fourier transform of the time series results. These peak frequencies, assumed to be the flutter frequencies, are also given in Table 10. The approximate flutter modes were then determined by correlating the FFT peaks to the Nextel natural frequencies from linear theory in Table 3.

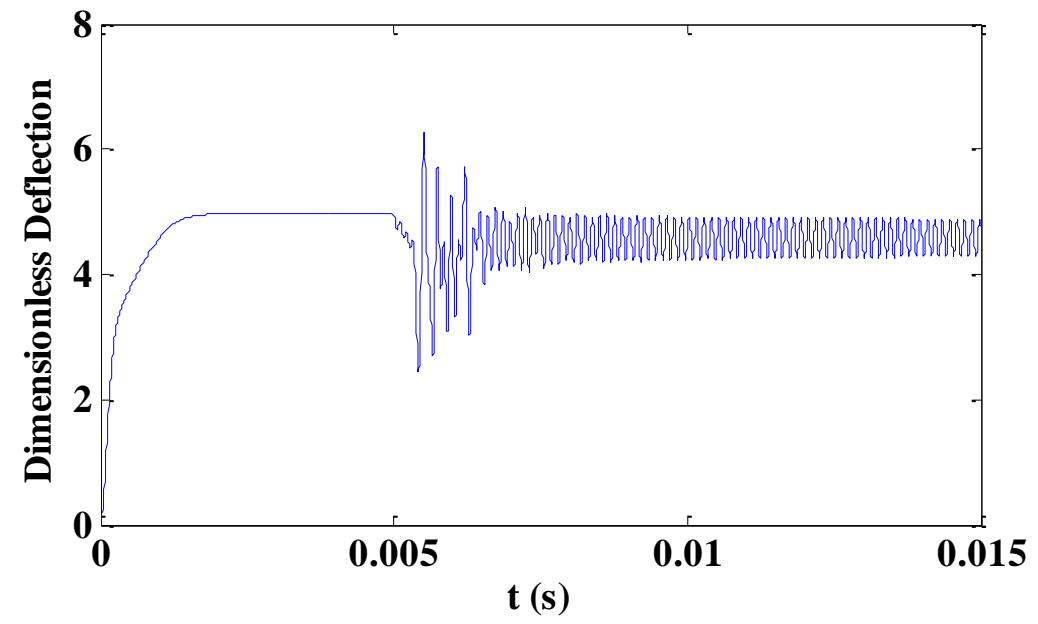

Figure 19. Nextel response (LCO) just above the flutter boundary, with $H / h=5$. The time history is sampled at a fixed location along the plate. Deflection has been normalized by the Nextel thickness.

The flutter mode shape in Figure 20 illustrates two important features of the imposed pressure model. First, the leading edge of the Nextel is compressed from the oncoming flow, but the pressure-imposed geometry is generally retained. Just beyond the flutter boundary, the trailing edge begins to flutter at a relatively small amplitude, which is indicated by the red circle.

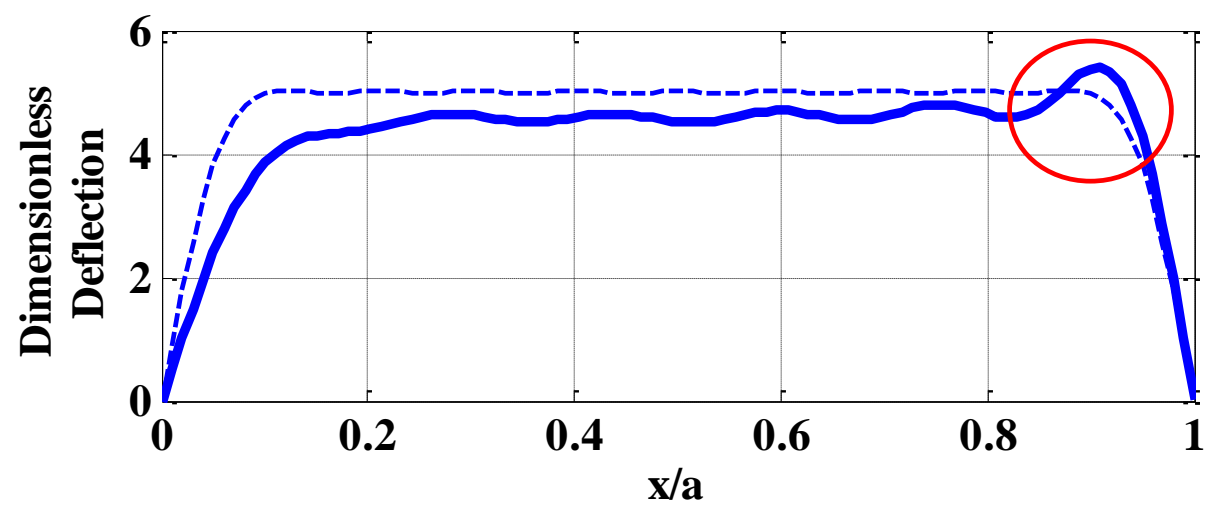

Figure 20. Flutter mode shape of the Nextel alone at an arbitrary time, with $H / h=5$. The dotted line indicates the pressure-imposed shape. Deflection has been normalized by the Nextel thickness. 


\section{Flutter of the 3-layer TPS.}

The flutter densities of the 3-layer TPS are lower than the Nextel alone, which was also observed in the parabolic curvature model. These results along with the peak response frequencies are given in Table 11. The limit cycle oscillations are also periodic and similar to the behavior seen in Figure 19. While the flutter modes in this case are generally the same as the Nextel alone, the peak response frequencies are significantly lower. This is consistent with the lower natural frequencies for the 3-layer TPS computed in the linear analysis. The flutter behavior in Figure 21 is characterized by significant static deflection of the initial pressure-imposed geometry, especially at the leading edge, while the dynamic oscillatory behavior is generally found towards the trailing edge.

Table 11: Flutter densities for the 3-layer TPS at different values of $H / h$, with $M=5$.

\begin{tabular}{|c|c|c|c|c|}
\hline $\mathbf{H} / \mathbf{h}$ & $\begin{array}{c}\text { Flutter density } \boldsymbol{\rho}_{\mathbf{f}} \\
\left(\mathbf{k g} / \mathbf{m}^{\mathbf{3}}\right)\end{array}$ & \# of streamwise modes & $\begin{array}{c}\text { Peak in FFT } \\
(\mathbf{r a d} / \mathbf{s})\end{array}$ & $\begin{array}{c}\text { Approx. Flutter } \\
\text { Modes* }^{*}\end{array}$ \\
\hline $\mathbf{0}$ & 0.5 & 4 & 1450 & 1,2 \\
\hline $\mathbf{1}$ & 1.4 & 6 & 2695 & 3,4 \\
\hline $\mathbf{2}$ & 3.2 & 8 & 5413 & 4,5 \\
\hline $\mathbf{3}$ & 5.2 & 12 & 10466 & 6,7 \\
\hline $\mathbf{4}$ & 7.8 & 14 & 13035 & 7,8 \\
\hline $\mathbf{5}$ & 10.5 & 16 & 14959 & 8,9 \\
\hline
\end{tabular}

*10 modes given to both the Nextel and Kevlar in the eigenvalue calculation

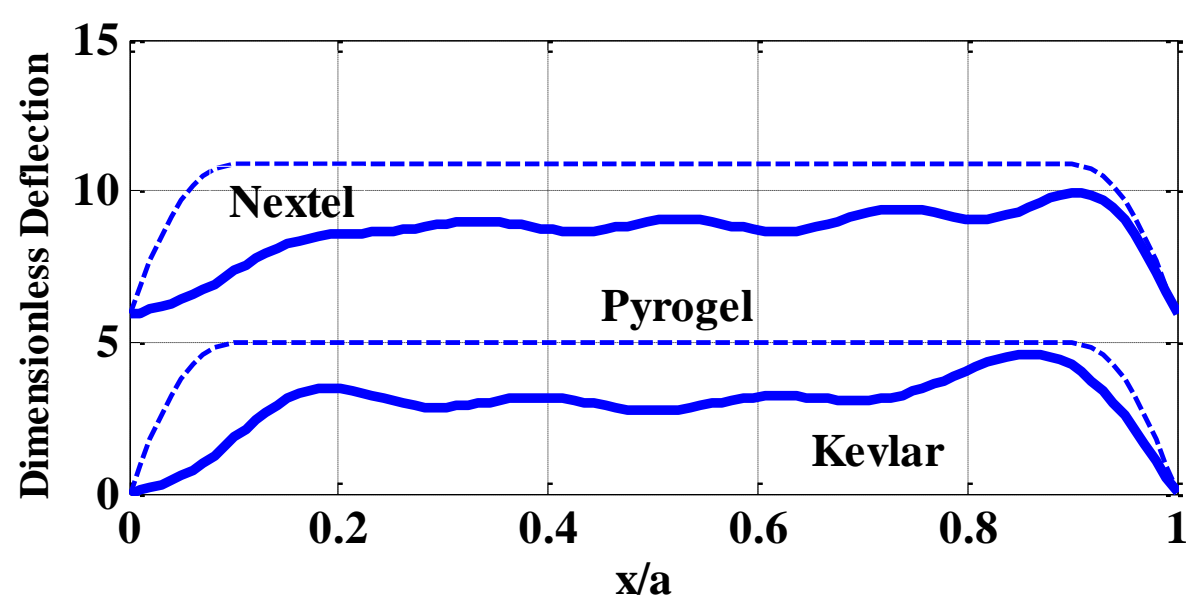

Figure 21. Flutter mode shape of the 3-layer TPS at an arbitrary time, with $H / h=5$. The dotted line indicates the pressure-imposed shape. Deflection has been normalized by the Nextel thickness.

\section{Initial Conditions}

As in the parabolic curvature model, no initial conditions are necessary in these simulations due to the initially imposed pressure. When additional initial conditions are specified, the computed flutter results are unchanged. It was also found that applying the static pressure and aerodynamic pressure at the same time $(t=0)$ produced the same results as delaying the aerodynamic pressure until the desired curvature was attained, as in Figure 19.

\section{Conclusion}

The flutter behavior of a NASA HIAD thermal protection system in its wind-tunnel test configuration has been evaluated in this paper. Specifically, the proposed TPS configuration consisting of Nextel 440-BF20, Pyrogel 6650, and Kapton Kevlar Laminate was studied. Three theoretical structural models were developed based on photographic observations of the experimental test setup as described in previous conference proceedings ${ }^{1,2}$. The piston theory hypersonic flow approximation was used for the aerodynamic pressure in all cases. 
The first structural model was a linear system of two-dimensional flat plates, which was solved using an eigenvalue analysis. With the Mach number fixed to a value of five, the flutter density parameters and flutter frequencies for various configurations were computed. It was determined that the Nextel outer layer governs the flutter behavior when coupled with the Pyrogel and Kevlar. The linear analysis also indicated that the TPS will flutter at Mach 5 with a flow density below that of air at sea level. Large in-plane loading of $1000 \mathrm{~N} / \mathrm{m}$ applied at all edges slightly increases the flutter density parameter, however, more realistic loading would have a negligible effect. Structural damping was also found to have little effect on the flutter.

The second structural model included the effect of initial parabolic curvature, which may arise from the restraint of the TPS test article at the boundaries. Von Karman's large deflection theory was used, resulting in a nonlinear system of equations. The limit cycle oscillations of the TPS in this configuration are chaotic, with large flutter amplitudes on the order of the curvature rise height. It was determined that the flutter density could either increase or decrease with increasing rise height depending on the nature of the curvature.

In the third structural model, curvature is imposed due to a static pressure from the rigid substructure, and the flutter behavior is significantly different. The effect of the static pressure is to increase significantly both the flutter frequency and flutter density, with periodic limit cycle response on the order of the Nextel thickness rather than the rise height. Plots of mode shapes indicated higher mode flutter at the trailing edge, rather than chaotic behavior seen in the parabolic curvature case. For both the parabolic and pressure-imposed curvature models, the 3-layer TPS has a noticeably lower flutter boundary than the Nextel alone for all nonzero rise heights. This indicates that the manner in which the TPS layers are stitched (or not stitched) will have an effect on the flutter behavior, but only if there is curvature present.

Further examination of the TPS article in its experimental test configuration is needed to determine which structural model is the most realistic. In any case, the preceding analysis may help to suggest the best method for flutter suppression in the wind-tunnel tests. Out of all the models, the effect of imposing a static pressure on the underside of the TPS had the greatest effect in raising the flutter boundary, while in-plane loading and structural damping had less of an effect. The linear analysis also indicated that clamped boundary conditions had higher flutter densities than simply supported conditions. This suggests that modifying the restraint bracket in the test setup to prevent rotation at the TPS edges would also raise the flutter boundary.

\section{Appendix}

\section{A. Modal Equations of Motion - Linear Flat Plate Model}

The modal equations resulting from the application of Lagrange's equations are given in this section. All integrals have been written in dimensionless form for convenient numerical integration. The dimensionless variables are $\xi=x / a$ and $\eta=y / b$.

$$
\begin{aligned}
& p^{2} \underbrace{\left\{m^{K E V} \hat{a}_{n} \int_{0}^{1} \int_{0}^{1} \varphi_{n}^{2} d \xi d \eta+\hat{a}_{n} m^{P Y R} \int_{0}^{1} \int_{0}^{1} \varphi_{n}^{2} d \xi d \eta+m^{P Y R} \sum_{r} \hat{b}_{r} \int_{0}^{1} \int_{0}^{1} \varphi_{n} \psi_{r} d \xi d \eta\right\}}_{M_{K E V}} \\
& +p \underbrace{\left\{2 \hat{a}_{n} \zeta \omega_{o}^{K E V} \int_{0}^{1} \int_{0}^{1} \varphi_{n}^{2} d \xi d \eta\right\}}_{C_{K E V}}+\left\{\frac{\hat{a}_{n} D^{K E V}}{a^{4}} \int_{0}^{1} \int_{0}^{1}\left(\frac{\partial^{2} \varphi_{n}}{\partial \xi^{2}}\right)^{2} d \xi d \eta+\frac{\hat{a}_{n} D^{K E V}}{b^{4}} \int_{0}^{1} \int_{0}^{1}\left(\frac{\partial^{2} \varphi_{n}}{\partial \eta^{2}}\right)^{2} d \xi d \eta\right. \\
& +\frac{2 \hat{a}_{n} v^{K E V} D^{K E V}}{a^{2} b^{2}} \int_{0}^{1} \int_{0}^{1}\left(\frac{\partial^{2} \varphi_{n}}{\partial \xi^{2}}\right)\left(\frac{\partial^{2} \varphi_{n}}{\partial \eta^{2}}\right) d \xi d \eta+\frac{2 \hat{a}_{n}\left(1-v^{K E V}\right) D^{K E V}}{a^{2} b^{2}} \int_{0}^{1} \int_{0}^{1}\left(\frac{\partial \varphi_{n}}{\partial \xi}\right)^{2}\left(\frac{\partial \varphi_{n}}{\partial \eta}\right)^{2} d \xi d \eta \\
& +\frac{\hat{a}_{n} N_{x}{ }^{K E V}}{a^{2}} \int_{0}^{1} \int_{0}^{1}\left(\frac{\partial \varphi_{n}}{\partial \xi}\right)^{2} d \xi d \eta+\frac{\hat{a}_{n} N_{y}{ }^{K E V}}{b^{2}} \int_{0}^{1} \int_{0}^{1}\left(\frac{\partial \varphi_{n}}{\partial \eta}\right)^{2} d \xi d \eta+\hat{a}_{n} k^{P Y R} \int_{0}^{1} \int_{0}^{1} \varphi_{n}^{2} d \xi d \eta \\
& \left.-k^{P Y R} \sum_{r} \hat{b}_{r} \int_{0}^{1} \int_{0}^{1} \varphi_{n} \psi_{r} d \xi d \eta\right\}=0
\end{aligned}
$$




$$
\begin{aligned}
& p^{2}\{\underbrace{N E X} \hat{b}_{r} \int_{0}^{1} \int_{0}^{1} \psi_{r}^{2} d \xi d \eta+b_{r} m^{P Y R} \int_{0}^{1} \int_{0}^{1} \psi_{r}^{2} d \xi d \eta+m^{P Y R} \sum_{n} a_{n} \int_{0}^{1} \int_{0}^{1} \varphi_{n} \psi_{r} d \xi d \eta\} \\
& +p\{\underbrace{\left\{2 \hat{b}_{r} \zeta \omega_{o}^{N E X} \int_{0}^{1} \int_{0}^{1} \psi_{r}^{2} d \xi d \eta+\hat{b}_{r} \rho_{\infty} a_{\infty} \int_{0}^{1} \int_{0}^{1} \psi_{r}^{2} d \xi d \eta\right\}}_{M_{N E X}} \\
& +\left\{\frac{\hat{b}_{r} D^{N E X}}{a^{4}} \int_{0}^{1} \int_{0}^{1}\left(\frac{\partial^{2} \psi_{r}}{\partial \xi^{2}}\right)^{2} d \xi d \eta+\frac{\hat{b}_{r} D^{K E V}}{b^{4}} \int_{0}^{1} \int_{0}^{1}\left(\frac{\partial^{2} \psi_{r}}{\partial \eta^{2}}\right)^{2} d \xi d \eta+\frac{2 \hat{b}_{r} \nu^{N E X} D^{N E X}}{a^{2} b^{2}} \int_{0}^{1} \int_{0}^{1}\left(\frac{\partial^{2} \psi_{r}}{\partial \xi^{2}}\right)\left(\frac{\partial^{2} \psi_{r}}{\partial \eta^{2}}\right) d \xi d \eta\right. \\
& +\frac{2 \hat{b}_{r}\left(1-v^{N E X}\right) D^{N E X}}{a^{2} b^{2}} \int_{0}^{1} \int_{0}^{1}\left(\frac{\partial \psi_{r}}{\partial \xi}\right)^{2}\left(\frac{\partial \psi_{r}}{\partial \eta}\right)^{2} d \xi d \eta+\frac{\hat{b}_{r} N_{x}^{N E X}}{a^{2}} \int_{0}^{1} \int_{0}^{1}\left(\frac{\partial \psi_{r}}{\partial \xi}\right)^{2} d \xi d \eta+\frac{\hat{b}_{r} N_{y}^{N E X}}{b^{2}} \int_{0}^{1} \int_{0}^{1}\left(\frac{\partial \psi_{r}}{\partial \eta}\right)^{2} d \xi d \eta \\
& +\underbrace{(A 2)}_{\left.K_{r} k^{P Y R} \int_{0}^{1} \int_{0}^{1} \psi_{r}^{2} d \xi d \eta-k^{P Y R} \sum_{n} \hat{a}_{n} \int_{0}^{1} \int_{0}^{1} \varphi_{n} \psi_{r} d \xi d \eta+\sum_{l} \frac{\hat{b}_{r} \rho_{\infty} a_{\infty} U}{a} \int_{0}^{1} \int_{0}^{1} \frac{\partial \psi_{l}}{\partial \xi} \psi_{r}(\xi) d \xi d \eta\right\}}
\end{aligned}
$$

The generalized forces, including aerodynamic pressure and structural damping are:

$$
\begin{aligned}
& Q_{n}=a b\left(2 \hat{a}_{n} \zeta \omega_{o}^{K E V} \int_{0}^{1} \int_{0}^{1} \varphi_{n}^{2} d \xi d \eta\right) \\
& Q_{r}=a b\left(\sum_{l} \frac{\hat{b}_{r} \rho_{\infty} a_{\infty} U}{a} \int_{0}^{1} \int_{0}^{1} \frac{\partial \psi_{l}}{\partial \xi} \psi_{r}(\xi) d \xi d \eta+\hat{b}_{r} \rho_{\infty} a_{\infty} \int_{0}^{1} \int_{0}^{1} \psi_{r}^{2} d \xi d \eta+2 \hat{b}_{r} \zeta \omega_{o}^{N E X} \int_{0}^{1} \int_{0}^{1} \psi_{r}^{2} d \xi d \eta\right)
\end{aligned}
$$

Thus, the mass, damping, and stiffness matrices in the eigenvalue problem (1.10) are:

$$
M=\left[\begin{array}{c}
M_{K E V} \\
M_{N E X}
\end{array}\right] \quad C=\left[\begin{array}{l}
C_{K E V} \\
C_{N E X}
\end{array}\right] \quad K=\left[\begin{array}{l}
K_{K E V} \\
K_{N E X}
\end{array}\right]
$$

\section{B. Modal Equations of Motion - Parabolic Curvature Model}

For brevity, the equations presented here are for a single (general) plate only, but the formulation can be easily extended to a 3-layer system by following the approach used for the linear flat plate model (though an eigenvalue analysis is not possible here). Using Galerkin integration, the expression for the modal coordinate of the Airy stress function is: 


$$
\begin{aligned}
& f_{k}=\left[\frac{E h}{\int_{0}^{1} \int_{0}^{1} \frac{\partial^{4} \gamma_{k}}{\partial \xi^{4}} \gamma_{k} d \xi d \eta+\left(\frac{a}{b}\right)^{4} \int_{0}^{1} \int_{0}^{1} \frac{\partial^{4} \gamma_{k}}{\partial \eta^{4}} \gamma_{k} d \xi d \eta+2\left(\frac{a}{b}\right)^{2} \int_{0}^{1} \int_{0}^{1} \frac{\partial^{4} \gamma_{k}}{\partial \xi^{2} \partial \eta^{2}} \gamma_{k} d \xi d \eta}\right] \times \\
& {\left[\left(\frac{a}{b}\right)^{2} \sum_{n} \sum_{i} a_{n} a_{i}\left(\int_{0}^{1} \int_{0}^{1} \frac{\partial^{2} \phi_{n}}{\partial \xi \partial \eta} \frac{\partial^{2} \phi_{i}}{\partial \xi \partial \eta} \gamma_{k} d \xi d \eta-\int_{0}^{1} \int_{0}^{1} \frac{\partial^{2} \phi_{n}}{\partial \xi^{2}} \frac{\partial^{2} \phi_{i}}{\partial \eta^{2}} \gamma_{k} d \xi d \eta\right)\right.} \\
& +H\left(\frac{a}{b}\right)^{2} \sum_{n} a_{n}\left(\int_{0}^{1} \int_{0}^{1} 2 \frac{\partial^{2} \phi_{n}}{\partial \xi \partial \eta} \frac{\partial^{2} \hat{z}}{\partial \xi \partial \eta} \gamma_{k} d \xi d \eta-\int_{0}^{1} \int_{0}^{1} \frac{\partial^{2} \phi_{n}}{\partial \eta^{2}} \frac{\partial^{2} \hat{z}}{\partial \xi^{2}} \gamma_{k} d \xi d \eta\right. \\
& \left.\left.-\int_{0}^{1} \int_{0}^{1} \frac{\partial^{2} \phi_{n}}{\partial \xi^{2}} \frac{\partial^{2} \hat{z}}{\partial \eta^{2}} \gamma_{k} d \xi d \eta\right)\right]
\end{aligned}
$$

The plate rise function has been non-dimensionalized using $z=H \hat{z}$. For convenience, let the large factor with $E h$ in the numerator be the quantity $C(k)$. Let the sum of the two integrals multiplying the product $a_{n} a_{i}$ be $I(n, i, k)$ and the sum of the three integrals multiplying $a_{n}$ be $J(n, k)$. Thus, $f_{k}$ can be written as

$$
f_{k}=C(k)\left[\left(\frac{a}{b}\right)^{2} \sum_{n} \sum_{i} a_{n} a_{i} I(n, i, k)+H\left(\frac{a}{b}\right)^{2} \sum_{n} a_{n} J(n, k)\right]
$$

This expression is then used in the expansion of the Airy function, along with the particular solution in Eq. (1.17). The generalized force due to aerodynamics and structural damping is:

$$
\begin{aligned}
& Q_{n}=a b\left[\rho_{\infty} a_{\infty} \dot{a}_{n} \int_{0}^{1} \int_{0}^{1} \phi_{n}^{2} d \xi d \eta+\frac{\rho_{\infty} a_{\infty} U_{\infty}}{a} \sum_{i} a_{i} \int_{0}^{1} \int_{0}^{1} \frac{\partial \phi_{i}}{\partial x} \phi_{n} d \xi d \eta\right. \\
& +\underbrace{\frac{\rho_{\infty} a_{\infty} U_{\infty} H}{a} \int_{0}^{1} \int_{0}^{1} \frac{\partial \hat{z}}{\partial x} \phi_{n} d \xi d \eta}_{*}+2 \zeta \omega_{0} \dot{a}_{n} \int_{0}^{1} \int_{0}^{1} \phi_{n}^{2} d \xi d \eta]
\end{aligned}
$$

The highlighted term in the generalized force expression is the static pressure loading due curvature, noted earlier as a critical factor in the flutter behavior of curved plates.

The equation of motion for this system is:

$$
\begin{aligned}
& m \ddot{a}_{n} \int_{0}^{1} \int_{0}^{1} \phi_{n}^{2} d \xi d \eta+\frac{1}{2 E h}\left(\frac{a}{b}\right)^{4} \sum_{k} R(k) C(k)^{2}\left(\sum_{i} \sum_{j} \sum_{s} a_{i} a_{j} a_{s}\{I(n, i, k) \times I(j, s, k)\}\right. \\
& \left.+H \sum_{i} \sum_{j} a_{i} a_{j}\{I(n, i, k) \times J(j, k)+J(n, k) \times I(i, j, k)\}+H^{2} \sum_{i} a_{i}\{J(n, k) \times J(i, k)\}\right) \\
& +\frac{1}{2 E h}\left(\frac{a}{b}\right)^{2} \sum_{k} L(k) C(k)\left(\sum_{i} a_{i} I(n, i, k)+H J(n, k)\right)+D a_{n} S(n)+\rho_{\infty} a_{\infty} \dot{a}_{n} \int_{0}^{1} \int_{0}^{1} \phi_{n}^{2} d \xi d \eta \\
& +\frac{\rho_{\infty} a_{\infty} U_{\infty}}{a} \sum_{i} a_{i} \int_{0}^{1} \int_{0}^{1} \frac{\partial \phi_{i}}{\partial x} \phi_{n} d \xi d \eta+\frac{\rho_{\infty} a_{\infty} U_{\infty} H}{a} \int_{0}^{1} \int_{0}^{1} \frac{\partial z}{\partial x} \phi_{n} d \xi d \eta+2 \zeta \omega_{0} \dot{a}_{n} \int_{0}^{1} \int_{0}^{1} \phi_{n}^{2} d \xi d \eta=0
\end{aligned}
$$


The additional variables $R(k), L(k)$, and $S(n)$ resulting from integral expressions in the stretching and bending energies are:

$$
\begin{aligned}
& R(k)=\left(\left(\frac{1}{a^{4}}\right) \int_{0}^{1} \int_{0}^{1}\left(\frac{\partial^{2} \gamma_{k}}{\partial \xi^{2}}\right)^{2} d \xi d \eta+\left(\frac{1}{b^{4}}\right) \int_{0}^{1} \int_{0}^{1}\left(\frac{\partial^{2} \gamma_{k}}{\partial \eta^{2}}\right)^{2} d \xi d \eta-2 v\left(\frac{1}{a^{2} b^{2}}\right) \int_{0}^{1} \int_{0}^{1} \frac{\partial^{2} \gamma_{k}}{\partial \xi^{2}} \frac{\partial^{2} \gamma_{k}}{\partial \eta^{2}} d \xi d \eta\right. \\
& \left.+2(1+v)\left(\frac{1}{a^{2} b^{2}}\right) \int_{0}^{1} \int_{0}^{1}\left(\frac{\partial^{2} \gamma_{k}}{\partial \xi \partial \eta}\right)^{2} d \xi d \eta\right) \\
& L(k)=\left\{\frac{2 \bar{N}_{y}}{a^{2}} \int_{0}^{1} \int_{0}^{1}\left(\frac{\partial^{2} \gamma_{k}}{\partial \xi^{2}}\right) d \xi d \eta+\frac{2 \bar{N}_{x}}{b^{2}} \int_{0}^{1} \int_{0}^{1}\left(\frac{\partial^{2} \gamma_{k}}{\partial \eta^{2}}\right) d \xi d \eta-2 v \frac{\bar{N}_{x}}{a^{2}} \int_{0}^{1} \int_{0}^{1}\left(\frac{\partial^{2} \gamma_{k}}{\partial \xi^{2}}\right) d \xi d \eta\right. \\
& -2 v \frac{\bar{N}_{y}}{b^{2}} \int_{0}^{1} \int_{0}^{1}\left(\frac{\partial^{2} \gamma_{k}}{\partial \eta^{2}}\right) d \xi d \eta+4(1+v) \frac{\bar{N}_{x y}}{a b} \int_{0}^{1} \int_{0}^{1}\left(\frac{\partial^{2} \gamma_{k}}{\partial \xi \partial \eta}\right) d \xi d \eta \\
& S(n)=\left(\left(\frac{1}{a^{4}}\right) \int_{0}^{1} \int_{0}^{1}\left(\frac{\partial^{2} \phi_{n}}{\partial \xi^{2}}\right)^{2} d \xi d \eta+\left(\frac{1}{b^{4}}\right) \int_{0}^{1} \int_{0}^{1}\left(\frac{\partial^{2} \phi_{n}}{\partial \eta^{2}}\right)^{2} d \xi d \eta+\left(\frac{1}{a^{2} b^{2}}\right) 2 v \int_{0}^{1} \int_{0}^{1}\left(\frac{\partial^{2} \phi_{n}}{\partial \xi^{2}}\right)\left(\frac{\partial^{2} \phi_{n}}{\partial \eta^{2}}\right) d \xi d \eta\right. \\
& \left.+2(1-v)\left(\frac{1}{a^{2} b^{2}}\right) \int_{0}^{1} \int_{0}^{1}\left(\frac{\partial^{2} \phi_{n}}{\partial \xi \partial \eta}\right)^{2} d \xi d \eta\right)
\end{aligned}
$$

\section{Modal Equations of Motion - Imposed Pressure Model}

The equations of motion for this case are also given by Eq. (A8), with the rise height $\mathrm{H}$ set to zero. This renders the plate initially flat, but the nonlinearity of the material is retained. Curvature will be imposed via a generalized force that corresponds to a pressure satisfying the static problem in Eq. (1.21). The expression for this generalized force is:

$$
\begin{aligned}
Q_{n}^{p} & =-\int_{0}^{1} \int_{0}^{1} p_{b} \alpha_{n} d \xi d \eta(a b) \\
& =-\frac{1}{2 E h}\left(\frac{a}{b}\right)^{4} \sum_{k} R(k) C(k)^{2}\left(\sum_{i} \sum_{j} \sum_{s} c_{i} c_{j} c_{s}\{I(n, i, k) \times I(j, s, k)\}\right) \\
& -\frac{1}{2 E h}\left(\frac{a}{b}\right)^{2} \sum_{k} L(k) C(k)\left(\sum_{i} c_{i} I(n, i, k)+H J(n, k)\right)-D c_{n} S(n)
\end{aligned}
$$

The mode shape functions $\alpha_{n}$ are the same functions given by the expansion in Eq. (1.20). Note that a single subscript is written in the equations of motion, but this implies summation over both streamwise and spanwise directions. The Fourier coefficients, expanded out in both directions, are: 


$$
\begin{aligned}
& c_{n m}=\left\{\frac{H}{p_{1}^{2}}\left[\frac{\left(\pi^{2} n^{2} p_{1}^{2}-2\right) \cos \left(n \pi p_{1}\right)}{\pi^{3} n^{3}}-\frac{2 p_{1}^{2} \cos \left(n \pi p_{1}\right)}{n \pi}+\frac{2}{\pi^{3} n^{3}}\right]+\frac{H}{n \pi}\left[\cos \left(n \pi p_{1}\right)-\cos \left(n \pi p_{2}\right)\right]\right. \\
& +\frac{H}{\left(1-p_{2}\right)^{2}}\left[\frac{\left(\pi^{2} n^{2}-2\right) \cos (n \pi)}{\pi^{3} n^{3}}-\frac{2 p_{2} \cos (n \pi)}{n \pi}+\frac{2\left(p_{2}+1\right)}{\pi^{3} n^{3}}+\frac{\left(2-\pi^{2} n^{2} p_{2}^{2}\right) \cos \left(n \pi p_{2}\right)}{\pi^{3} n^{3}}+\frac{2 p_{2}^{2} \cos \left(n \pi p_{2}\right)}{n \pi}\right. \\
& \left.\left.+\frac{2\left(1-2 p_{2}\right) \cos \left(n \pi p_{2}\right)}{\pi^{3} n^{3}}\right]\right\} \times\left\{\frac{1}{p_{1}^{2}}\left[\frac{\left(\pi^{2} m^{2} p_{1}^{2}-2\right) \cos \left(m \pi p_{1}\right)}{\pi^{3} m^{3}}-\frac{2 p_{1}^{2} \cos \left(m \pi p_{1}\right)}{m \pi}+\frac{2}{\pi^{3} m^{3}}\right]\right. \\
& +\frac{1}{m \pi}\left[\cos \left(m \pi p_{1}\right)-\cos \left(m \pi p_{2}\right)\right]+\frac{1}{\left(1-p_{2}\right)^{2}}\left[\frac{\left(\pi^{2} m^{2}-2\right) \cos (m \pi)}{\pi^{3} m^{3}}-\frac{2 p_{2} \cos (m \pi)}{m \pi}+\frac{2\left(p_{2}+1\right)}{\pi^{3} m^{3}}\right. \\
& \left.\left.+\frac{\left(2-\pi^{2} m^{2} p_{2}^{2}\right) \cos \left(m \pi p_{2}\right)}{\pi^{3} m^{3}}+\frac{2 p_{2}^{2} \cos \left(m \pi p_{2}\right)}{m \pi}+\frac{2\left(1-2 p_{2}\right) \cos \left(m \pi p_{2}\right)}{\pi^{3} m^{3}}\right]\right\}
\end{aligned}
$$

\section{References}

${ }^{1}$ J. A. Del Corso, F. M. Cheatwood, W. E. Bruce, S. J. Hughes and A. M. Calomino, "Advanced HighTemperature Flexible TPS for Inflatable Aerodynamic Decelerators," 21st AIAA Aerodynamic Decelerator Systems Technology Conference and Seminar, Vol. 1, AIAA, Dublin, 2011, pp. 139-161.

${ }^{2}$ S. J. Hughes, J. S. Ware, J. A. Del Corso and R. A. Lugo, "Deployable Aeroshell Flexible Thermal Protection System Testing," 20th AIAA Aerodynamic Decelerator Systems Technology Conference, Vol 1.,AIAA, Seattle, 2009, pp. 291-311. 2,3 .

${ }^{3}$ E. H. Dowell, Aeroelasticity of Plates and Shells, Noordhoff International Publishing, Leyden, 1975, Chaps.

${ }^{4}$ E. H. Dowell, "Nonlinear Flutter of Curved Plates," AIAA Journal, vol. 7, no. 3, pp. 424-431, 1969.

53M, "Ceramic Textiles and Composites Bulletin," 3M, Saint Paul, MN, 1996.

${ }^{6}$ The Dupont Company, "Kevlar Aramid Fiber Technical Guide," 30 October 2001. [Online]. Available: www.dupont.com. [Accessed 20 June 2012].

${ }^{7}$ Microstructured Materials Group, "Silica Aerogels," Lawrence Berkeley National Laboratory, 1 January 2012. [Online]. Available: http://energy.lbl.gov/ecs/aerogels/sa-physical.html. [Accessed 20 June 2012].

${ }^{8}$ E. H. Dowell, "Nonlinear Oscillations of a Fluttering Plate," AIAA Journal, vol. 4, no. 7, pp. 1267-1275, 1966. 